\title{
THE FREUDENTHAL-SPRINGER-TITS CONSTRUCTIONS REVISITED
}

\author{
BY \\ KEVIN MCCRIMMON
}

In [3] some constructions of exceptional Jordan algebras due to $\mathrm{H}$. Freudenthal, T. A. Springer, and J. Tits were carried over to quadratic Jordan algebras (as in [4]) of arbitrary characteristic. The question was left open whether the Tits Constructions yielded all exceptional finite-dimensional central simple algebras in characteristic 2 (it was known that they do for characteristics $\neq 2$ ). In this paper we settle this question in the affirmative. This result completes the structure theory for finite-dimensional quadratic Jordan algebras.

The Tits Constructions as given in [3] involve the construction of a norm form. To prove that all the exceptional algebras arise from these constructions we need to show that all such algebras have a suitable norm form. This necessitates a slight detour in $\S \S 1$ and 2 to verify that Jordan algebras in characteristic 2 have generic norms with the same properties as in the other characteristics. In $\S 3$ we define the centroid for quadratic Jordan algebras and the corresponding notion of central simple algebras. In the next section we establish certain conditions under which a central simple Jordan algebra remains simple upon extension of the base field. In $\S 5$ we apply these results to show that every exceptional finite-dimensional central simple algebra is a form of the 27-dimensional exceptional algebra $\mathfrak{S}\left(\mathfrak{E}_{3}\right)$ (i.e. becomes $\mathfrak{S}_{\mathfrak{S}}\left(\mathfrak{C}_{3}\right)$ upon suitable extension of the base field). In the final section we show that the Tits Constructions yield precisely all the exceptional finite-dimensional central simple Jordan algebras. Our proofs will be valid for all characteristics.

1. Generically algebraic algebras. Except when we explicitly state otherwise (as in §3), all algebras and vector spaces will be taken over a field $\Phi$ of arbitrary characteristic. We do not assume finite-dimensionality. We will need to make use of the generic norm and its basic properties. These concepts are defined for arbitrary power-associative algebras [5], but quadratic Jordan algebras are not power-associative in the usual sense-since they are not even defined in terms of a bilinear product, we cannot talk of "associativity" of powers.

We are forced to use a more general definition. A power algebra $\mathfrak{P}$ is a vector space $\mathfrak{X}$ together with a family of polynomial maps $p_{n}$ of $\mathfrak{X}$ into itself (the power maps) for $n=0,1,2, \ldots$ such that

(i) each $p_{n}$ is homogeneous of degree $n$;

Received by the editors March 4, 1969 and, in revised form, September 2, 1969.

Copyright (C) 1970, American Mathematical Society 
(ii) $p_{0} \neq 0$;

(iii) $p_{1}$ is the identity map.

Thus $p_{0}$ is a constant, $p_{0}(x) \equiv c$, where the unit element $c$ is nonzero, and $p_{1}(x)=x$ for all $x$. If the field $\Phi$ is finite, we agree that a "polynomial map" $p$ is a formal polynomial map rather than just a set-theoretic map, so that for any extension $\Omega$ of $\Phi$ there is a unique extension $p_{\Omega}$ of $p$ to $\mathfrak{X}_{\Omega}$; if $\Phi$ is infinite, formal and settheoretic maps are equivalent. These conventions guarantee that for each extension $\Omega$ of the base field we can extend $\mathfrak{P}$ to a power algebra $\mathfrak{P}_{\Omega}$ on $\mathfrak{X}_{\Omega}$. For example, if $\mathfrak{A}$ is an ordinary unital linear algebra on $\mathfrak{X}$ then $\mathfrak{X}$ and the maps $p_{n}(x)=x^{n}$ constitute the power algebra associated with $\mathfrak{A}$. If $\mathfrak{\Im}$ is a unital quadratic Jordan algebra on $\mathfrak{X}$ then $\mathfrak{X}$ and the power maps $p_{0}(x)=c, p_{1}(x)=x, p_{n+2}(x)=U_{x} p_{n}(x)$ define the power algebra associated with $\mathfrak{\Im}$. A map $\theta: \mathfrak{P} \rightarrow \tilde{\mathfrak{P}}$ is a homomorphism of power algebras if $\theta\left(p_{n}(x)\right)=\tilde{p}_{n}(\theta(x))$ for all $n$ and all $x$ (hence in particular $\theta(c)=\tilde{c}$ ).

We say a power algebra $\mathfrak{P}$ is power-associative if for each $x$ the linear map $\hat{x}: \Phi[\lambda] \rightarrow \mathfrak{X}$ given by $\hat{x}\left(\lambda^{n}\right)=p_{n}(x)$ satisfies

(iv) $\hat{x}$ is a homomorphism of power algebras;

(v) the kernel of $\hat{x}$ is an ideal in $\Phi[\lambda]$.

$\mathfrak{W}$ is strictly power-associative if all extensions $\mathfrak{B}_{\Omega}$ are power-associative. If for $q(\lambda) \in \Phi[\lambda]$ we let $q(x)$ denote $\hat{x}(q(\lambda))$ then (iv) reduces to $q^{n}(x)=p_{n}(q(x))$ for all $n$ and all $q(\lambda)$, or equivalently

(iv) $^{\prime}(p \circ q)(x)=p(q(x))$ for all $p(\lambda), q(\lambda) \in \Phi[\lambda]$. From this it is clear that if $\mathfrak{P}$ satisfies (iv) and $\Phi$ is infinite then all $\mathfrak{H}_{\Omega}$ will satisfy (iv). It is not clear whether (v) necessarily remains valid upon extension if $\Phi$ is infinite, and thus whether a powerassociative algebra over an infinite field is necessarily strictly power-associative.

If we let $\Phi[x]$ denote the subspace of $\mathfrak{X}$ spanned by the $p_{n}(x)$ 's (so $c$ and $x$ belong to $\Phi[x]$ ) then condition (v) guarantees that we have a linear bijection of $\Phi[\lambda] / \operatorname{Ker} \hat{x}$ onto $\Phi[x]$ so that $\Phi[x]$ inherits the structure of a commutative associative algebra. This induced structure is compatible with the original, since (iv) implies the power maps $y \rightarrow y^{n}$ in the induced structure coincide with the original $y \rightarrow p_{n}(y)$ for $y \in \Phi[x]$. If $\mathfrak{A}$ is a power-associative (or strictly power-associative) linear algebra then the associated power algebra is power-associative (or strictly power-associative). If $\mathfrak{\Im}$ is a quadratic Jordan algebra the associated power algebra will not in general be power-associative: there are examples of elements $x$ satisfying $x^{2}=0$ but $x^{3} \neq 0$. (Note that only (v) is violated; by Macdonald's Theorem [7] (iv) is always satisfied in a quadratic Jordan algebra. Also, (v) can only be violated in characteristic 2.) We do have power-associativity in one important case, namely

Proposition 1. If the unital quadratic Jordan algebra $\mathfrak{I}$ contains no nonzero absolute zero divisors (so $U_{z}=0 \Rightarrow z=0$ ) then the associated power algebra is powerassociative.

Proof. As we remarked above, (iv) is always satisfied by Macdonald's Theorem. For (v), clearly $\operatorname{Ker} \hat{x}$ is a linear subspace, and if $p(\lambda) \in \Phi[\lambda], q(\lambda) \in \operatorname{Ker} \hat{x}$ then 
$r(\lambda)=p(\lambda) q(\lambda)$ has $U_{r(x)}=U_{p(x)} U_{q(x)}$ (again by Macdonald's Theorem [7]) $=0$ (since $q(\lambda) \in \operatorname{Ker} \hat{x}$ means $q(x)=0)$; thus $r(x)$ is an absolute zero divisor, and by hypothesis this implies $r(x)=0$. Thus $r(\lambda) \in \operatorname{Ker} \hat{x}$ and $\operatorname{Ker} \hat{x}$ is an ideal in $\Phi[\lambda]$.

With these definitions, the results of [5, pp. 533-538], (see also [1, pp. 221-229]) carry over mutatis mutandis to power algebras. A strictly power-associative power algebra $\mathfrak{B}$ is generically algebraic if it satisfies some monic polynomial relation $f_{x}(\lambda)=\sum f_{i}(x) \lambda^{i}$ where the $f_{i}$ are polynomial functions on $\mathfrak{X}$ (thus $f_{x}(x)=\sum f_{i}(x) p_{i}(x)$ $=0$ for all $x$ ). The monic polynomial $m_{x}(\lambda)=\sum m_{1}(x) \lambda^{i}$ of least degree which is satisfied by $\mathfrak{P}$ is the generic minimum polynomial of $\mathfrak{P}$, and the degree of $m_{x}(\lambda)$ is called the generic degree of 1 . The generic norm is $N(x)=(-1)^{m} m_{0}(x)$ and the generic trace is $T(x)=-m_{m-1}(x)$. We have

(1) $m_{i}(x)$ is a form of degree $m-i$,

(2) $m_{i}(c)=(-1)^{m-i}\left(\begin{array}{c}m \\ i\end{array}\right)$ for $c$ the unit,

(3) $\left.\partial_{c} m_{i-1}\right|_{x}=-i m_{i}(x)$,

(4) $N(y z)=N(y) N(z)$ if $y, z \in \Phi[x]$.

(3) was not proven in [5], but it follows easily by differentiating the minimum equation, $\left.\partial_{c}\left\{\sum m_{i}(x) p_{i}(x)\right\}\right|_{x}=0$.

As in [5, p. 540], [1, p. 229] we can define the generic discriminant $\delta$ of a generically algebraic power algebra $\mathfrak{B}$. This is a form of degree $\frac{1}{2} m(m+1)$ where $m$ is the generic degree, such that $\delta(x)=0$ if and only if $m_{x}(\lambda)$ has repeated roots. We say $\mathfrak{P}$ is generically unramified if $\delta(x)$ is not identically zero on $\mathfrak{B}$; this happens if and only if there are $m$ nonzero orthogonal idempotents in $\mathfrak{A}_{\Omega}$ for $\Omega$ the algebraic closure of $\Phi$. (We say $\left\{e_{i}\right\}$ are orthogonal idempotents if the powers of any element $x=\sum \alpha_{i} e_{i}$ are given by $x^{n}=\sum \alpha_{i}^{n} e_{i}$ and this remains true in all extensions $\Omega$ of $\Phi-$ for distinct $\alpha_{i} \neq 0$ this means $\Phi[x]$ is isomorphic as power algebra and as associative algebra to a direct sum $\Phi \oplus \cdots \oplus \Phi$ of $m$ copies of the base field $\Phi$.)

For generically algebraic Jordan algebras we have the further results

(5) $N(x) \neq 0$ if and only if $x$ is invertible,

(6) $N\left(U_{x} y\right)=N(x)^{2} N(y)$,

(7) $T(x \circ y, z)=T(x, y \circ z)$,

(8) $T\left(x^{2}, x\right)=T\left(x^{3}\right)$,

(9) $T(x, x)=T\left(x^{2}\right)$,

where

$$
T(x, y)=-\left.\partial_{x} \partial_{y} \log N\right|_{c}
$$

satisfies $T(x)=T(x, c)$. (5) and (6) follows as in [5], while (8) and (9) can be derived from (4) because if $N$ admits associative composition on $\Phi[x]$ then $T$ is an associative form on $\Phi[x][8$, formula (5), p. 76]. Similarly the argument in [8, pp. 75-76] can be used to derive (7).

Professor T. A. Springer has asked whether the set of squares is dense in the split exceptional Jordan algebra $\mathfrak{S}\left(\mathfrak{C}_{3}\right)$. This is true in characteristic $\neq 2$, for then the differential of the map $x \rightarrow x^{2}$ at the unit element is $2 I$ and hence invertible. 
This argument is valid for any sort of nonassociative linear algebra with unit. However, if we are willing to restrict ourselves to unramified generically algebraic power algebras we can avoid any restriction of the characteristic.

PROPOSITION 2. Let $\mathfrak{i}$ be an unramified generically algebraic power algebra over $\Phi, p(\lambda) \in \Phi[\lambda]$ a polynomial of degree $n>0$. If $F: \mathfrak{P} \rightarrow \Phi$ is a polynomial function of degree $m$ such that $F(p(x))=0$ for all $x$ then $F=0$ if $|\Phi|>n m$. Consequently, if $\Phi$ is infinite the set of $p(x)$ 's is dense in '‥

Proof. Since $F \circ p$ is a polynomial function of degree $\leqq n m$, if $F(p(x))=0$ for all $x \in \mathfrak{B}$ then $F(p(x))=0$ for all $x$ in any extension $\mathfrak{B}_{\Omega}$ by our assumption on $|\Phi|$. If $\delta(x) \neq 0$ then the generic minimum polynomial $m_{x}(\lambda)$ (and hence the minimum polynomial of $x$ in $\Phi[x])$ has distinct roots, so $x=\sum \alpha_{i} e_{i}$ for $\alpha_{i}$ in the algebraic closure $\Omega$ of $\Phi$ and $e_{i} \in \Omega[x]$ are orthogonal idempotents. The equations $\alpha_{i}=p\left(\beta_{i}\right)$ can be solved for $\beta_{i} \in \Omega$ since $\Omega$ is algebraically closed and $p(\lambda)$ is of degree $>0$. Then $y=\sum \beta_{i} e_{i} \in \Omega[x]$ has $p(y)=\sum p\left(\beta_{i}\right) e_{i}=\sum \alpha_{i} e_{i}=x$. Thus any $x$ with $\delta(x) \neq 0$ is of the form $p(y)$, and the set of $p(y)$ 's contains the dense set of $x$ 's with $\delta(x) \neq 0$ (dense because it is nonempty by our assumption that $\mathfrak{P}$ is unramified). Since $F$ vanishes on this dense set we must have $F=0$.

Taking $p(\lambda)=\lambda^{2}$ and $\mathfrak{B}$ the power algebra associated with $\mathfrak{S}_{\mathfrak{C}}\left(\mathfrak{S}_{3}\right)$ over an infinite field we see the set of squares is dense. Here $\mathfrak{S}_{\mathfrak{S}}\left(\mathfrak{C}_{3}\right)$ is generically algebraic of degree 3 , and unramified because it contains 3 nonzero orthogonal idempotents.

2. Algebras of degree three. Throughout this section we assume $\mathfrak{\Im}$ is a unital quadratic Jordan algebra which is generically algebraic of degree 3 , with minimum equation

(10) $x^{3}-T(x) x^{2}+S(x) x-N(x) c=0$

where $T$ is linear, $S$ quadratic, and $N$ cubic. We introduce a quadratic adjoint map by

(11) $x^{\#}=x^{2}-T(x) x+S(x) c$.

By power-associativity we see $x x^{\#}=N(x) c$, so $x^{\#}=N(x) x^{-1}$ whenever $x$ is invertible (which is whenever $N(x) \neq 0$ by (5)). As usual we let $x \times y=(x+y) \#-x^{\#}$ $-y^{\#}$ be the associated bilinear product.

THEOREM 1. If $\Im$ is a unital quadratic Jordan algebra which is generally algebraic of degree three then $\mathfrak{\Im}=\mathfrak{\Im}(N, \#, c)$ for $N$ the generic norm, \# the adjoint, and $c$ the unit.

Proof. We must first show $N$, \#, $c$ satisfy the conditions necessary to be able to construct $\Im(N, \#, c)[3$, p. 499]:

(i) $\quad x^{\# \#}=N(x) x$,

(ii) $\quad T\left(x^{\#}, y\right)=\left.\partial_{y} N\right|_{x}$,

(12) (iii) $c \times y=T(y) c-y$,

(iv) $c^{\#}=c$,

(v) $\quad N(c)=1$. 
It suffices to prove (i) on the Zariski-dense set where $N(x) \neq 0$; there $x^{\sharp \#}=N(x) x^{-1}$ has $N\left(x^{\#}\right)=N(x)^{3} N\left(x^{-1}\right)=N(x)^{2} \neq 0$, so $x^{\# \#}=N\left(x^{\#}\right) x^{\#-1}=N(x)^{2}\left\{N(x) x^{-1}\right\}^{-1}$ $=N(x)^{2} N(x)^{-1}\left(x^{-1}\right)^{-1}=N(x) x$. The proof of (ii) is quite involved, so we postpone it for a moment. Property (v) follows from (2), hence (iv) $c^{\#}=N(c) c^{-1}=c$, while $c \times y=c \circ y-T(c) y-T(y) c+\left(\left.\partial_{c} S\right|_{x}\right) c$ (linearizing (11)) $=2 y-3 y-T(y) c+2 T(y) c$ (since $T(c)=3$ by (2), $\left.\partial_{c} S\right|_{x}=2 T(x)$ by (3)) $=T(y) c-y$ establishes (iii).

We now show that 2 times the relation (ii) holds,

(13) $2 T\left(x^{\#}, y\right)=\left.2 \partial_{y} N\right|_{x}$.

Taking logarithmic derivatives of $(6), N\left(U_{x} z\right)=N(x)^{2} N(z)$, we get

and

$$
\left.\partial_{y} \log \left\{N\left(U_{x} z\right)\right\}\right|_{x}=\left.N\left(U_{x} z\right)^{-1} \partial_{y} N\left(U_{x} z\right)\right|_{x}=\left.N\left(U_{x} z\right)^{-1} \partial_{U(x, y) z} N\right|_{U(x) z}
$$

$$
\left.\partial_{y} \log \left\{N(x)^{2} N(z)\right\}\right|_{x}=\left.2 \partial_{y} \log N\right|_{x}=\left.2 N(x)^{-1} \partial_{y} N\right|_{x} .
$$

Setting $z=x^{-2}$, so $U_{x} z=c$ and $\left\{x x^{-2} y\right\}=x^{-1} \circ y$ gives $\left.2 N(x)^{-1} \partial_{y} N\right|_{x}=\left.\partial_{x^{-1}{ }^{\circ} y} N\right|_{c}$ $=T\left(x^{-1} \circ y\right)=2 T\left(x^{-1}, y\right)$ (linearizing $T\left(x^{2}\right)=T(x, x)$ by (9)). Thus $\left.2 \partial_{y} N\right|_{x}=$ $2 T\left(N(x) x^{-1}, y\right)=2 T\left(x^{\#}, y\right)$ on the dense set where $N(x) \neq 0$, so it holds everywhere.

We next prove 3 times (ii) holds,

(14) $3 T\left(x^{\sharp}, y\right)=\left.3 \partial_{y} N\right|_{x}$

We have $T(x, y)=\left.\left.\partial_{x} N\right|_{c} \cdot \partial_{y} N\right|_{c}-\left.\partial_{x} \partial_{y} N\right|_{c}=T(x) T(y)-\left.\partial_{y} \partial_{c} N\right|_{x}\left(\left.\partial_{x} \partial_{y} N\right|_{z}=\left.\partial_{x} \partial_{y} \partial_{z} N\right|_{c}\right.$ is symmetric in $x, y, z$ since $N$ is of degree 3$)=T(x) T(y)-\partial_{y} S(x)\left(\left.\partial_{c} N\right|_{x}=S(x)\right.$ by (3)), so

(15) $\left.\partial_{y} S\right|_{x}=T(x) T(y)-T(x, y)$.

Setting $y=x$, Euler's differential equation gives

(16) $2 S(x)=T(x)^{2}-T(x, x)=T(x)^{2}-T\left(x^{2}\right)$.

We have $T\left(x^{\sharp}, x\right)=T\left(x^{2}, x\right)-T(x) T(x, x)+S(x) T(c, x)$ (by $\left.(11)\right)=T\left(x^{3}\right)-T(x) T\left(x^{2}\right)$ $+S(x) T(x)$ (by (8), (9)) $=N(x) T(c)$ (taking traces of (10)). By (2) we get the case $y=x$ of (ii),

(17) $T\left(x^{\#}, x\right)=3 N(x)$.

Linearizing gives $T\left(x^{\#}, y\right)=-T(x \times y, x)+\left.3 \partial_{y} N\right|_{x}$. Now

$$
T(x \times y, x)=T(x \circ y, x)-T(x) T(y, x)-T(y) T(x, x)+\left(\left.\partial_{y} S\right|_{x}\right) T(c, x)
$$

(using the linearized form of (11))

$$
=T(x \circ x, y)-T(x) T(x, y)-T(y) T\left(x^{2}\right)+T(x)\{T(x) T(y)-T(x, y)\}
$$

(by (7), (9), (15))

$$
\begin{aligned}
& =2 T\left(x^{2}, y\right)-2 T(x) T(x, y)+T(y)\left\{T(x)^{2}-T\left(x^{2}\right)\right\} \\
& =2\left\{T\left(x^{2}, y\right)-T(x) T(x, y)+S(x) T(y)\right\}
\end{aligned}
$$

(by $(16))=2 T\left(x^{\#}, y\right)$ (by (11)). Thus the previous formula reduces to $T\left(x^{\#}, y\right)$ $=-2 T\left(x^{\#}, y\right)+\left.3 \partial_{y} N\right|_{x}$, which is just (14). Subtracting (13) from (14) gives (ii).

Thus $\Im(N, \#, c)$ can be defined. We note that $y=c$ in (ii) gives

(18) $S(x)=T\left(x^{\#)}\right.$

by (3), hence linearization yields

(19) $\left.\partial_{y} S\right|_{x}=T(x \times y)$ 
and, comparing with (15), the very useful formula

(20) $T(x \times y)+T(x, y)=T(x) T(y)$.

Linearizing (10) and noting that $x^{3}=U_{x} x$, the $U$-operator in $\mathfrak{I}$ is expressible as

$$
\begin{aligned}
U_{x} y & =-U_{x, y} x+T(y) x^{2}+T(x) x \circ y-S(x) y-\left(\left.\partial_{y} S\right|_{x}\right) x+\left(\left.\partial_{y} N\right|_{x}\right) c \\
& =-x^{2} \circ y+T(y) x^{2}+T(x) x \circ y-T\left(x^{\#}\right) y-T(x \times y) x+T\left(x^{\#}, y\right) c
\end{aligned}
$$

(using (18), (19), (12.ii) and $\{x x y\}=x^{2} \circ y$ by $[4$, p. 1073, (14)])

$$
\begin{aligned}
= & -\left\{x^{\#}+T(x) x-T\left(x^{\#}\right) c\right\} \circ y+T(y)\left\{x^{\#}+T(x) x-T\left(x^{\#}\right) c\right\} \\
& +T(x) x \circ y-T\left(x^{\#}\right) y-\{T(x) T(y)-T(x, y)\} x+T\left(x^{\#}, y\right) c
\end{aligned}
$$

(using (11), (20))

$$
\begin{aligned}
& =-x^{\#} \circ y+T\left(x^{\#}\right) y+T(y) x^{\#}-\left\{T(y) T\left(x^{\#}\right)-T\left(x^{\#}, y\right)\right\} c+T(x, y) x \\
& =T(x, y) x-x^{\#} \times y
\end{aligned}
$$

by (19), (20), and the linearized version of (11). But this is precisely how the $U$ operator is defined in $\Im(N, \#, c)$. Thus $\mathfrak{\Im}(N, \#, c)$ and $\mathfrak{\Im}$ both have the same module structure, the same unit element, and the same $U$-operator, so they are the same quadratic Jordan algebra.

REMARK. Note that by linearizing (12) (ii) we get $T(x \times y, z)=\left.\partial_{y} \partial_{z} N\right|_{x}$, so as we have noted before

(21) $T(x \times y, z)=\left.\partial_{x} \partial_{y} \partial_{z} N\right|_{c}$ is symmetric in $x, y, z$.

This will be of use later. We also recall the adjoint formulas [3, pp. 496, 501] deduced from (12) (i), (12) (ii)

(i) $\quad x^{\#} \times(x \times y)=N(x) y+T\left(x^{\#}, y\right) x$,

(ii) $\quad x \times\left(x^{\#} \times y\right)=N(x) y+T(x, y) x^{\#}$,

(iii) $\quad N\left(x^{\#)}=N(x)^{2}\right.$,

(iv) $\quad N(x \times y)+N(x) N(y)=T\left(x^{\#}, y\right) T\left(x, y^{\#}\right)$.

3. The centroid. In this section we allow $\Phi$ to be an arbitrary commutative associative ring with unit. The centroid $\Gamma(\mathfrak{\Im} / \Phi)$ of a quadratic Jordan algebra $\mathfrak{\Im}$ over $\Phi$ is the set of all $\Phi$-endomorphisms $T$ of $\mathfrak{\Im}$ such that

$$
\begin{aligned}
& \text { (i) } T U_{x}=U_{x} T, \quad T V_{x}=V_{x} T \text {, } \\
& \text { (ii) } U_{T x}=T^{2} U_{x}, \quad(T x)^{2}=T^{2} x^{2}
\end{aligned}
$$

for all $x \in \mathfrak{J}$ (if $1 \in \mathfrak{\Im}$ then the second condition of each pair is superfluous). When the ring of scalars $\Phi$ is understood we just write $\Gamma(\Im)$. If we regard such a $T$ as a "scalar" $\gamma$ we see that $U_{x}, V_{x}$ are $\Gamma$-linear transformations by (i), and that $x \rightarrow U_{x}$, $x \rightarrow x^{2}$ are $\Gamma$-quadratic maps $\left(U_{\gamma x}=\gamma^{2} U_{x},(\gamma x)^{2}=\gamma^{2} x^{2}, U_{\gamma x, y}=\gamma U_{x, y}\right.$ by $\{\gamma x z y\}$ $=V_{y, z}(\gamma x)=\gamma V_{y, z} x=\gamma\{x z y\}$, and $V_{\gamma x}=\gamma V_{x}$ by $\left.(\gamma x) \circ y=V_{y}(\gamma x)=\gamma V_{y} x=\gamma(x \circ y)\right)$. If $\Gamma$ were a commutative ring then $\mathfrak{\Im}$ would be a $\Gamma$-algebra in a natural way. Note that always $\Phi I \subset \Gamma$. We say $\mathfrak{\Im}$ is central over $\Phi$ if $\Gamma=\Phi$. If $\Gamma$ is commutative then 
the centroid of $\Im / \Gamma$ and $\Im / \Phi$ coincide with $\Gamma$ (hence the same holds for any $\Phi \subset \Omega \subset \Gamma$ ) since all $\gamma$ in $\Gamma$ are $\Gamma$-linear if $\Gamma$ is commutative.

(24) $\Gamma(\Im / \Gamma)=\Gamma(\Im / \Phi)$ if $\Gamma(\Im / \Phi)$ is commutative.

In this case $\mathfrak{\Im}$ is central when regarded as an algebra over $\Gamma$.

If $\frac{1}{2} \in \Phi$ then $\Gamma$ is just the set of $T$ satisfying $T V_{x}=V_{x} T$, the usual centroid for the linear algebra defined by the product $x \circ y=V_{x} y$ (this follows since the $U$ structure $U_{x} y=\frac{1}{2}\left\{V_{x}^{2}-V_{x^{2}}\right\} y$ and squaring operation $x^{2}=\frac{1}{2} x \circ x$ can be defined in terms of the product $x \circ y$ ).

For a linear algebra $\mathfrak{A}$ the centroid is always a ring and is commutative if $\mathfrak{A}$ has unit 1 or if $\mathfrak{A}^{2}=\mathfrak{A}$. The situation is less satisfying for quadratic algebras.

THEOREM 2. If the quadratic Jordan algebra $\mathfrak{\Im}$ has $\Gamma(\Im)$ commutative then $\Gamma(\Im)$ is a ring; conversely, if $\mathfrak{\Im}=U_{\mathfrak{J}} \mathfrak{\Im}$ or $\mathfrak{\Im}^{2}=\mathfrak{\Im}$ (in particular, if $\mathfrak{\Im}$ has a unit) then commutativity is necessary for $\Gamma(\mathfrak{\Im})$ to be a ring. If $\mathfrak{\Im}=\mathfrak{\Im} \circ \mathfrak{\Im}$ or $\mathfrak{\Im}=\{\mathfrak{\Im} \mathfrak{\Im} \mathfrak{\Im}\}$ or if $\mathfrak{\Im}$ has zero extreme radical then $\Gamma$ is commutative.

Proof. Suppose $S, T \in \Gamma$. Then $S+T$ and $S T$ clearly commute with all $U_{x}$ and $V_{x}$ since $S$ and $T$ do, so they satisfy condition (i). The sticky point is (ii):

$$
\begin{aligned}
U_{(S+T) x} & =U_{S x+T x}=U_{S x}+U_{S x, T x}+U_{T x} \\
& =S^{2} U_{x}+S T U_{x, x}+T^{2} U_{x}=\left(S^{2}+2 S T+T^{2}\right) U_{x} \\
((S+T) x)^{2} & =(S x+T x)^{2}=(S x)^{2}+S x \circ T x+(T x)^{2} \\
& =S^{2} x^{2}+S T x \circ x+T^{2} x^{2}=\left(S^{2}+2 S T+T^{2}\right) x^{2}
\end{aligned}
$$

while $(S+T)^{2}=S^{2}+S T+T S+T^{2}$, so $S+T \in \Gamma$ if and only if

(i) $[S, T] U_{x}=0$;

(ii) $[S, T] x^{2}=0$

for all $x \in \mathfrak{\Im}$. Similarly $U_{S T x}=S^{2} T^{2} U_{x},(S T x)^{2}=S^{2} T^{2} x^{2}$ while $(S T)^{2}=S T S T$, so $S T \in \Gamma$ if and only if

(iii) $S[S, T] T U_{x}=0$;

(iv) $S[S, T] T x^{2}=0$.

Clearly if $\Gamma$ is commutative then by (i)-(iv) $T+S$ and $S T$ belong to $\Gamma$ and $\Gamma$ is a ring. Also, if $U_{\mathfrak{J}} \mathfrak{\Im}=\mathfrak{\Im}$ or $\mathfrak{\Im}^{2}=\mathfrak{\Im}$ then the commutativity is necessary by (i) or (ii). It is easy to see that $\Gamma$ is commutative if $\mathfrak{\Im} \circ \mathfrak{\Im}=\mathfrak{I}$ or $\{\mathfrak{I} \mathfrak{I}\}=\mathfrak{J}$ since

$$
\begin{aligned}
S T(x \circ y) & =S(x \circ T y)=S x \circ T y=T(S x \circ y)=T S(x \circ y), \\
S T\left\{\begin{array}{lll}
x & y & z
\end{array}\right\} & =\left\{\begin{array}{lll}
S x & y & T z
\end{array}\right\}=T S\left\{\begin{array}{lll}
x & y & z
\end{array}\right\} .
\end{aligned}
$$

Finally, we prove $[S, T] \mathfrak{\Im} \subset \mathbb{Z}=\left\{z \in \mathfrak{S} \mid U_{z}=V_{z}=U_{z, x}=0\right.$ for all $\left.x\right\}=$ the extreme radical. We have $U_{(S T-T S) x}=U_{S T x}-U_{S T x, T S x}+U_{T S x}=S^{2} U_{T x}-S U_{T x, S x} T+U_{S x} T^{2}=$ $S^{2} U_{x} T^{2}-S^{2} U_{x, x} T^{2}+S^{2} U_{x} T^{2}=0$ (being careful always to take $S$ and $T$ out of terms like $U_{S T x}$ on different sides); similarly $V_{(S T-T S) x}=S V_{x} T-S V_{x} T=0$ and $U_{(S T-T S) x, y}=S U_{x, y} T-S U_{x, y} T=0$ for all $x$ and $y$, so that $[S, T] x \in Z$.

For the case of simple algebras we have the usual

TheOREM 3 (SCHUR's Lemma). If $\mathfrak{\Im}$ is simple then $\Gamma(\Im)$ is a field. 
Proof. The extreme radical 3 is an ideal, and $8 \neq \mathfrak{s}$ since $\mathfrak{F}$ is not a trivial algebra (with all products zero), so by simplicity $3=0$. Hence $\Gamma(\mathfrak{J})$ is a commutative ring by Theorem 2 . All that remains is to show that each $T \neq 0$ in $\Gamma$ is bijective (note that if $T$ is in $\Gamma$ then so is $T^{-1}$ ).

We first show $\Re=$ Range $T$ is an ideal in $\mathfrak{F}$. $\Re$ is an outer ideal $\left(U_{\mathfrak{I}} \Re \subset \Re\right.$, $\left.V_{\mathfrak{J}} \mathfrak{R} \subset \mathfrak{R}\right)$ by (i), and it is an inner ideal $\left(U_{\mathfrak{R}} \mathfrak{F} \subset \mathfrak{R}, \mathfrak{R}^{2} \subset \mathfrak{R}\right)$ since for any $y=T z \in \mathfrak{R}$ we have $U_{y} \mathfrak{\Im}=U_{T z} \mathfrak{\Im}=T^{2} U_{z} \mathfrak{\Im} \subset T \mathfrak{\Im}=\Re$ and $y^{2}=(T z)^{2}=T^{2} z^{2} \in T \mathfrak{\Im}=\Re$. Thus $\Re$ is an ideal.

If $\Re=0$ then $T=0$; otherwise $\Re=\mathfrak{I}$ and $T$ is surjective. If $\mathfrak{g}$ had a unit we could argue similarly to show $\mathfrak{R}=\operatorname{Ker} T$ is an ideal; however, a simple algebra need not be unital, so we will have to use a different argument. We will show $\Omega^{\prime}$ is contained in the extreme radical 8 if $T$ is surjective, hence $8=0$ implies $\Re=0$ and $T$ is bijective. So let $T$ (and hence $T^{2}$ ) be surjective, and consider $z \in \mathfrak{R}: T z=0$. We have $U_{z} \mathfrak{\Im}=U_{z} T^{2} \mathfrak{J}=U_{T z} \mathfrak{\Im}=0, V_{z} \mathfrak{\Im}=V_{z} T \mathfrak{J}=V_{T z} \mathfrak{\Im}=0$, and $U_{z, x} \mathfrak{\Im}=U_{z, x} T \mathfrak{\Im}=U_{T z, x} \mathfrak{\Im}=0$ so that $U_{z}=V_{z}=U_{z, x}=0$ for all $x$ and $z \in 3$.

Note that simplicity is independent of the ring of scalars: $\mathfrak{F}$ is simple as an algebra over $\Phi$ if and only if it is simple as a ring (i.e. an algebra over $\boldsymbol{Z}$ ). Clearly if $\mathfrak{\Im}$ contains no proper $Z$-ideals it contains no proper $\Phi$-ideals, and conversely if it contains no proper $\Phi$-ideals then for any nonzero $Z$-ideal $\Re, \Phi \Re$ is a nonzero $\Phi$-ideal, hence $\Phi \mathfrak{\Re}=\mathfrak{\Im}$, hence $\mathfrak{\Im}=U_{\mathfrak{J}} \mathfrak{\Im}=U_{\Phi \Omega} \mathfrak{I}=U_{\mathfrak{\Omega}} \Phi^{2} \mathfrak{\Im} \subset U_{\Re} \mathfrak{T} \subset \mathfrak{N}$ if $\mathfrak{\Re}$ is a $Z$-ideal, so $\mathfrak{N}=\mathfrak{\Im}$. In this case (indeed, whenever the centroid of $\mathfrak{\Im} / \boldsymbol{Z}$ is commutative) the centroid is also independent of the ring of scalars:

(25) $\Gamma(\mathfrak{J} / \boldsymbol{Z})=\Gamma(\mathfrak{\Im} / \Phi)$ if $\mathfrak{\Im}$ is simple.

In the case of unital linear algebras we identify the elements $\gamma$ of the centroid $\Gamma$ with the elements $\gamma 1$ of the algebra by ineans of $\gamma=L_{\gamma 1}$. For unital quadratic algebras there is no analogue of left multiplication (unless $\frac{1}{2} \in \Phi$ ), so we cannot identify the transformations with the elements. However, we still like to think of the elements of $\Gamma$ as having many of the properties of elements of $\Im$. For example, if $\sigma$ is an automorphism of $\mathfrak{s}$ we can apply it to elements of the centroid just as we can apply it to elements of the algebra.

Proposition 3. The mapping $T \rightarrow T^{\sigma}=\sigma \circ T \circ \sigma^{-1}$ defines a representation of the automorphism group Aut (Э) of $\Im$ as a group of automorphisms of the centroid $\Gamma / \Phi$; the kernel of the representation is the subgroup of $\Gamma$-linear automorphisms. The representation on $\Gamma$ commutes with the representation on $\mathfrak{\Im}$ in the sense that

$$
(T x)^{\sigma}=T^{\sigma} x^{\sigma} \quad(T \in \Gamma, x \in \mathfrak{\Im}, \sigma \in \text { Aut (Э)). }
$$

Proof. $T^{\sigma} x^{\sigma}=\left(\sigma \circ T \circ \sigma^{-1}\right) x^{\sigma}=(\sigma \circ T) x=(T x)^{\sigma}$ defines the action of $\sigma$ on $T$. Thus $T^{\sigma} U_{x^{\sigma}} y^{\sigma}=T^{\sigma}\left(U_{x} y\right)^{\sigma}=\left(T U_{x} y\right)^{\sigma}=\left(U_{x} T y\right)^{\sigma}=U_{x^{\sigma}}(T y)^{\sigma}=U_{x^{\sigma}} T^{\sigma} y^{\sigma}$ shows $T^{\sigma} U_{z}=U_{z} T^{\sigma}$ for all $z=x^{\sigma}$; similarly $T^{\sigma} V_{z}=V_{z} T^{\sigma}$, so $T^{\sigma}$ satisfies (23) (i). For (23) (ii) we have $U_{T^{\sigma} x^{\sigma}} y^{\sigma}=U_{(T x)^{\sigma}} y^{\sigma}=\left(U_{T x} y\right)^{\sigma}=\left(T^{2} U_{x} y\right)^{\sigma}=\left(T^{\sigma}\right)^{2} U_{x^{\sigma}} y^{\sigma}$ so $U_{T^{\sigma} z}=\left(T^{\sigma}\right)^{2} U_{z}$ for all $z=x^{\sigma}$, and similarly $\left(T^{\sigma} z\right)^{2}=\left(T^{\sigma}\right)^{2} z^{2}$. Thus $T^{\sigma}$ belongs to $\Gamma$. Clearly $T^{\sigma \pi}=\left(T^{\sigma}\right)^{\pi}$ (beware: $\sigma \pi=\pi \circ$ เ $)$ and $(T \circ S)^{\sigma}=T^{\sigma} \circ S^{\sigma},(T+S)^{\sigma}=T^{\sigma}+S^{\sigma},(\alpha T)^{\sigma}=\alpha T^{\sigma}$ for $\alpha \in \Phi$, so 
$T \rightarrow T^{\sigma}$ is a representation. $T^{\sigma}=T$ for all $T$ if and only if $T x^{\sigma}=T^{\sigma} x^{\sigma}=(T x)^{\sigma}$ for all $T$ in $\Gamma$, i.e. $\sigma$ is $\Gamma$-linear.

The outer centroid $\Gamma_{0}(\mathfrak{S})=\Gamma_{0}(\mathfrak{\Im} / \Phi)$ is the set of $\Phi$-linear transformations on $\mathfrak{\Im}$ which commute with all $U_{x}$ and $V_{x}$; it is just the centralizer of the multiplication

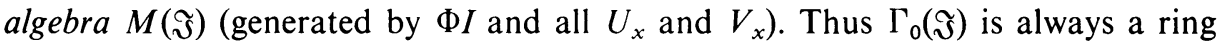
(not necessarily commutative) containing the centroid, but in general there is no way of making $\mathfrak{\Im}$ into a $\Gamma_{0}$-algebra. We say $\mathfrak{J}$ is outer-central if $\Gamma_{0}(\mathfrak{\Im})=\Phi$. This is stronger than just being central, though if $\frac{1}{2} \in \Phi$ then $\Gamma_{0}=\Gamma$ and the two notions coincide.

An easy calculation shows

Proposition 4. If $\mathfrak{\Im}=\oplus \mathfrak{\Im}_{i}$ is a direct sum of unital algebras $\mathfrak{\Im}_{i}$ then $\Gamma(\Im)=$ $\oplus \Gamma\left(\mathfrak{\Im}_{i}\right)$ and $\Gamma_{0}(\mathfrak{\Im})=\oplus \Gamma_{0}\left(\mathfrak{\Im}_{i}\right)$. If $\mathfrak{\Im}^{(u)}$ is an isotope of $\mathfrak{\Im}$ then $\Gamma\left(\mathfrak{\Im}^{(u)}\right)=\Gamma(\mathfrak{\Im})$ and $\Gamma_{0}\left(\mathfrak{J}^{(u)}\right)=\Gamma_{0}(\mathfrak{\Im})$.

(We need the units $1_{i} \in \mathfrak{\Im}_{i}$ so the projections $U_{1_{i}}$ of $\mathfrak{\Im}$ on $\mathfrak{\Im}_{i}$ commute with $\Gamma$ and $\Gamma_{0}$; the result for isotopes follows from the symmetry of isotopy [4, p. 1076] and the formulas $U_{x}^{(u)}=U_{x} U_{u}$ and $M\left(\mathfrak{\Im}^{(u)}\right)=M(\mathfrak{\Im})$.)

Proposition 5. If $\mathfrak{\Im}$ is an algebra over a field $\Phi$ then for every extension $\Omega$ of $\Phi$ we have

(i) $\Gamma_{0}\left(\Im_{\Omega}\right)=\Gamma_{0}(\Im)_{\Omega}$;

(ii) $\Gamma\left(\Im_{\Omega}\right) \supset \Gamma(\Im)_{\Omega}, \quad \Gamma(\Im)=\Gamma\left(\Im_{\Omega}\right) \cap \operatorname{End}_{\Phi}(\Im)$;

(iii) $\Gamma_{0}(\mathfrak{\Im})=\Gamma(\Im)$ if and only if $\Gamma_{0}\left(\mathfrak{\Im}_{\Omega}\right)=\Gamma\left(\Im_{\Omega}\right)$.

Proof. Since the $U_{x}$ for $x$ in $\mathfrak{\Im}$ span both $U_{\mathfrak{I}}$ (over $\Phi$ ) and $U_{\mathfrak{I}_{\Omega}}$ (over $\Omega$ ) we have (i). $T \in \operatorname{End}_{\Phi}(\mathfrak{\Im})$ satisfies (23) (i), (ii) on $\mathfrak{\Im}$ if and only if its extension to $\mathfrak{\Im}_{\Omega}$ satisfies them on $\mathfrak{\Im}_{\Omega}$, so $\Gamma(\Im)=\Gamma\left(\Im_{\Omega}\right) \cap \operatorname{End}_{\Phi}(\Im)$ (interpreting this in the natural way) and hence $\Gamma(\Im)_{\Omega} \subset \Gamma\left(\Im_{\Omega}\right)$, establishing (ii). Finally, if $\Gamma_{0}(\mathfrak{I})=\Gamma(\mathfrak{\Im})$ then $\Gamma_{0}\left(\Im_{\Omega}\right)$ $=\Gamma_{0}(\Im)_{\Omega}=\Gamma(\Im)_{\Omega} \subset \Gamma\left(\Im_{\Omega}\right)$ by (i) and (ii), and since we always have $\Gamma_{0}\left(\Im_{\Omega}\right) \supset \Gamma\left(\Im_{\Omega}\right)$ we see $\Gamma_{0}\left(\Im_{\Omega}\right)=\Gamma\left(\Im_{\Omega}\right)$. Conversely, if $\Gamma_{0}\left(\Im_{\Omega}\right)=\Gamma\left(\Im_{\Omega}\right)$ then $\Gamma(\Im)=\Gamma\left(\Im_{\Omega}\right) \cap \operatorname{End}_{\Phi}(\Im)$ $=\Gamma_{0}\left(\mathfrak{\Im}_{\Omega}\right) \cap \operatorname{End}_{\Phi}(\mathfrak{\Im})=\Gamma_{0}(\mathfrak{\Im})_{\Omega} \cap \operatorname{End}_{\Phi}(\mathfrak{\Im})=\Gamma_{0}(\mathfrak{\Im})$.

We compute the centroid and outer centroid for an important class of algebras.

Proposition 6. If $\mathfrak{D}$ is a unital alternative algebra with involution $*$, $\mathfrak{D}_{0}$ a subspace of *-symmetric elements of the nucleus containing 1 and such that $d \mathfrak{D}_{0} d^{*} \subset \mathfrak{D}_{0}$ for all $d \in \mathfrak{D}$, then the Jordan matrix algebra $\mathfrak{S}=\mathfrak{S}_{\mathfrak{C}}\left(\mathfrak{D}_{n}, \mathfrak{D}_{0}, \gamma\right)$ for $n \geqq 3$ (regarded as an algebra over $Z$ ) has $\Gamma(\Im)=\Gamma_{0}(\Im)=\Omega I$ where $\Omega$ is the set of elements $\omega$ in the center of $\mathfrak{D}$ such that $\omega \mathfrak{D}_{0} \subset \mathfrak{D}_{0}$.

Proof. Since $\mathfrak{S}\left(\mathfrak{D}_{n}, \mathfrak{D}_{0}, \gamma\right)$ is isomorphic to an isotope of $\mathfrak{S}_{\mathfrak{C}}\left(\mathfrak{D}_{n}, \mathfrak{D}_{0}\right)$ [4, p. 1077], and since $\Gamma$ and $\Gamma_{0}$ are independent of isotopy by Proposition 4, we need only consider $\mathfrak{\Im}=\mathfrak{S}\left(\mathfrak{D}_{n}, \mathfrak{D}_{0}\right)$.

$D$ can be regarded as an algebra with involution over the commutative algebra $\Omega$ (note $\Omega \subset \mathscr{D}_{0}$ so $\omega^{*}=\omega$ for all $\omega \in \Omega$ ); it is clear that all mappings in $\Omega I$ (all "scalar multiplications" by elements of $\Omega$ ) belong to the centroid, $\Omega I \subset \Gamma(\Im)$. 
Conversely, suppose $T$ belongs to $\Gamma_{0}(\Im)$. Then it commutes with the Peirce projections relative to the idempotents $e_{i}=1[i i]$ (here $x[i i]=x e_{i i}$ for $x \in \mathfrak{D}_{0}$, $x[i j]=x e_{i j}+x^{*} e_{j i}$ for $x \in \mathfrak{D}$ if $i \neq j$ where the $e_{i j}$ are the usual matrix units), so the Peirce spaces $\mathfrak{I}_{i j}=\mathfrak{D}[i j](i \neq j)$ and $\mathfrak{I}_{i i}=\mathfrak{I}_{0}[i i]$ are invariant under $T: T(x[i j])$ $=T_{i j}(x)[i j]$ for linear maps $T_{i i}: \mathfrak{D}_{0} \rightarrow \mathfrak{D}_{0}$ and $T_{i j}: \mathfrak{D} \rightarrow \mathfrak{D}$. If $i, j, k \neq$ then $T(x[i j] \circ y[j k])=(T x[i j]) \circ y[j k]=x[i j] \circ(T y[j k])$ and the formula $x[i j] \circ y[j k]$ $=x y[i k]$ gives $T_{i k}(x y)=T_{i j}(x) y=x T_{j k}(y)$. Taking $x=1$ or $y=1$ we see $T_{i k}=T_{i j}=T_{j k}$ for $i, j, k \neq \quad$; by our assumption $n \geqq 3$ this implies all $T_{i j}$ for $i \neq j$ have a common value $T_{0}$, where $T_{0}(x)=t x=x t$ for $t=T_{0}(1)$. Similarly $T(x[i i] \circ y[i j])=(T x[i i]) \circ y[i j]$ for $x \in \mathfrak{D}_{0}, y \in \mathfrak{D}$ gives $T_{i i}=T_{i j}=T_{0}$. But then $t=T_{0}(1)=T_{i i}(1) \in \mathcal{D}_{0}$ implies $t$ is in the nucleus, so $t x=x t$ for all $x$ guarantees $t$ is in the center, and $T_{i i}\left(\mathfrak{D}_{0}\right) \subset \mathfrak{D}_{0}$ implies $t \mathfrak{D}_{0} \subset \mathfrak{D}_{0}$ and $t \in \Omega$. Thus $T$ is just scalar multiplication by $t \in \Omega$, and $T \in \Omega I$.

This shows $\Gamma_{0}(\mathfrak{\Im}) \subset \Omega I \subset \Gamma(\mathfrak{\Im})$, whence equality $\Gamma_{0}(\mathfrak{\Im})=\Omega I=\Gamma(\mathfrak{\Im})$.

4. Simplicity under extension. Our structure theory tells us what the central simple algebras over an algebraically closed field look like, so if we knew that a central simple algebra $\mathfrak{\Im}$ over $\Phi$ remained simple when we passed to the algebraic closure $\Omega$ we would know that $\mathfrak{\Im}$ was a form of one of the standard algebras. In this section we will establish conditions under which simplicity will be preserved under extension.

EXAmPLE. Simplicity is not always preserved. Let $\Omega$ be a nonperfect field of characteristic 2, $\Omega_{0}=\Omega^{2}$ the (proper) subfield of squares. Then by Proposition 6 $\mathfrak{I}=\mathfrak{S}\left(\Omega_{n}, \Omega_{0}\right)$ for $n \geqq 3$ is a central simple Jordan algebra over $\Omega_{0}$. We claim $\mathfrak{I}_{\Omega}=\Omega \otimes_{\Omega_{0}} \mathfrak{\Im}$ is not simple, or even semisimple. Thus extension may not even preserve semisimplicity, in sharp contrast to the case of central simple linear algebras. In fact, if $\omega \in \Omega$ but $\omega \notin \Omega_{0}$ then for $i \neq j$ the element $z=\omega \otimes 1[i j]$ $-1 \otimes \omega[i j]$ is nonzero (we are tensoring over $\Omega_{0}$ ) and yet $U_{z}=0$, so $\Im_{\Omega}$ contains absolute zero divisors, hence is not semisimple [9, p. 678].

The difficulty is that simplicity of $\mathfrak{\Im}$ is not equivalent to irreducibility of $\mathfrak{\Im}$ under the multiplication algebra $M(\Im)$, as it is in the case of linear algebras. The invariant subspaces of $M(\mathfrak{\Im})$ are precisely the outer ideals, and an algebra without proper ideals may have proper outer ideals. (In the example $\mathfrak{g}\left(\Omega_{n}, \Omega_{0}\right)$ above the space $\mathfrak{i}$ of all matrices with zeros down the diagonal forms a proper outer ideal.) We say $\mathfrak{J}$ is outer-simple if it has no proper outer ideals-this is stronger than simplicity, though the concepts coincide in characteristic $\neq 2$.

THEOREM 4. If $\mathfrak{J}$ is outer-simple and outer-central over $\Phi$ then so is any extension $\mathfrak{\Im}_{\Omega}=\Omega \otimes_{\Phi} \mathfrak{\Im}$.

Proof. To say that there are no proper outer ideals and that $\Gamma_{0}(\Im)=\Phi$ means that $M(\mathfrak{J})$ acts irreducibly on $\mathfrak{\Im}$ with centralizer $\Phi$. We must show that $M\left(\Im_{\Omega}\right)=M(\Im)_{\Omega}$ acts irreducibly on $\Im_{\Omega}$ (hence $\mathfrak{\Im}_{\Omega}$ is outer-simple) with centralizer $\Omega$ (hence $\Im_{\Omega}$ is outer-central). But this is a consequence of the well-known associative result 
LEMma 1 [10, P. 292, THEOREM 3]. If $\mathfrak{A}$ is an associative algebra over a field $\Phi$, $\mathfrak{M}$ a left $\mathfrak{A}$-module such that the centralizer of $\mathfrak{A}$ on $\mathfrak{M}$ is just $\Phi$, then for any extension $\Omega \supset \Phi$ the centralizer of $\mathfrak{A}_{\Omega}$ on $\mathfrak{M}_{\Omega}$ is just $\Omega$. If $\mathfrak{M}$ is faithful or irreducible, so is $\mathfrak{M}_{\Omega}$.

The proof is an application of the Jacobson Density Theorem.

Proposition 7. Let $\mathfrak{D}$ be a composition algebra with nondegenerate trace form over a field $\mathfrak{D}_{0}=\Omega$. Then the Jordan matrix algebra $\mathfrak{S}_{(}\left(\mathfrak{D}_{n}, \mathfrak{D}_{0}, \gamma\right)$ for $n \geqq 2$ is outersimple as a ring (i.e. as an algebra over $Z$ ).

Proof. Again we may assume $\mathfrak{I}=\mathfrak{S}\left(\mathfrak{D}_{n}, \mathfrak{D}_{0}\right)$ by passing to an isotope, since outer ideals in $\mathfrak{I}$ and its isotopes coincide. Let $\mathfrak{I}$ be a nonzero outer $Z$-ideal. As in the proof of Proposition 6 , $\mathfrak{I}$ has the Peirce decomposition $\mathfrak{\Im}=\bigoplus_{i, j=1}^{n} \mathfrak{I}_{i j}$ relative to the diagonal idempotents. $\mathscr{N}$ is invariant under the Peirce projections $E_{i i}=U_{e_{i}}, E_{i j}=U_{e_{i}, e,}$ and hence $\mathfrak{\Re}=\oplus \mathfrak{\Re}_{i}$, for $\Re_{i j}=\Re \cap \Im_{i j}$. We first show some $\mathfrak{\Re}_{i j} \neq 0$ for $i \neq j$. Since $\mathfrak{N} \neq 0$ either some $\mathfrak{\Re}_{i j} \neq 0$ or some $\mathfrak{N}_{i i} \neq 0$, and if $x[i i] \in \mathfrak{\Re}_{i i}$ for $x \neq 0$ in $\mathscr{D}_{0}$ then $x \mathfrak{D}[i j]=x[i i] \circ \mathfrak{D}[i j] \subset \mathfrak{\Omega}_{i j}$ with $x \mathfrak{D} \neq 0$ since $x \in \mathfrak{D}_{0}=\Omega$ is invertible. Next, if $\mathfrak{\Re}_{i j} \neq 0$ we show $e_{i}, e_{j} \in \mathfrak{R}$. We have $d[i j] \in \mathfrak{\Re}_{i j}$ for some $d \neq 0$, so by nondegeneracy of the trace form there is $d^{\prime} \in \mathfrak{D}$ with $t\left(d, d^{\prime}\right)=1$. Hence $\mathfrak{R}$ contains $d[i j] \circ d^{\prime}[j i]=t\left(d, d^{\prime}\right)\left(e_{i}+e_{j}\right)=e_{i}+e_{j}$ and thus both $e_{i}$ and $e_{j}$. Once $e_{i}$ belongs to $\Re$ so do all $\mathfrak{\Im}_{i j}=e_{i} \circ \mathfrak{\Im}_{i j}$, hence by the above all of the $e_{j}$, hence all of the $\Im_{j k}$ for $j \neq k$. If $t(d)=1$ then $t(\omega d)=\omega$ for any $\omega \in \Omega$, so $\omega\left(e_{i}+e_{j}\right)=1[i j] \circ \omega d[j i]$ $\in \mathfrak{N}$, hence $\omega e_{i} \in \mathfrak{K}, \Omega e_{i} \subset \mathfrak{N}_{i i}$, and $\mathfrak{N}=\mathfrak{\Im}$.

PROPOSITION 8. If $\mathfrak{\Im}=\oplus \mathfrak{\Im}_{i}$ is a direct sum of unital algebras such that every outer ideal in $\mathfrak{\Im}_{i}$ is an ideal, then every outer ideal in $\mathfrak{\Im}$ is an ideal.

(If $\mathfrak{K}$ is outer then $\mathfrak{N}$ is invariant under the projections $U_{1_{i}}$ of $\mathfrak{I}$ on $\mathfrak{I}_{i}$, hence $\mathfrak{N}=\oplus \mathfrak{R}_{i}$ for $\mathfrak{R}_{i}$ outer in $\mathfrak{R}$.)

5. Simplicity under extension for exceptional algebras. Here we want to establish that for exceptional algebras, at least, extensions always preserve simplicity.

Recall that $\mathfrak{\Im}$ is special if and only if the natural homomorphism su: $\mathfrak{J} \rightarrow \operatorname{su}(\mathfrak{I})^{+}$ of $\mathfrak{\Im}$ into its special universal envelope su $(\mathfrak{\Im})$ is injective [4, pp. 1073-1074]. If $\mathfrak{J}$ is exceptional then su is not injective; if $\mathfrak{\Im}$ is also simple then su must be zero. Thus every exceptional simple Jordan algebra is purely exceptional in the sense that su $(\mathfrak{)})=0$. If $\mathfrak{\Im}$ is purely exceptional, so is any extension $\mathfrak{\Im}_{\Omega}$ and any homomorphic image $\overline{\mathfrak{I}}=\mathfrak{\Im} / \mathfrak{\Re}\left(\mathfrak{\Re}\right.$ an ideal) since su $\left(\mathfrak{\Im}_{\Omega}\right)=\operatorname{su}(\mathfrak{\Im})_{\Omega}$ and su $(\mathfrak{\Im} / \mathfrak{I})=\mathrm{su}(\mathfrak{\Im}) / \mathfrak{\Re}^{\prime}$ where $\mathfrak{\Re}^{\prime}$ is the ideal in su (Э) generated by the image of $\mathfrak{\Re}$.

First we recall the standard

Lemma 2 [10, P. 295, Lemma 2]. If $\mathfrak{X}$ is a vector space over a field $\Phi, \Omega$ a Galois extension of $\Phi$, then any subspace $\mathfrak{B}$ of $\mathfrak{X}_{\Omega}$ invariant under the natural action of the Galois group $G(\Omega / \Phi)$ on $\mathfrak{X}_{\Omega}=\Omega \otimes \mathfrak{X}$ is of the form $\mathfrak{Y}_{\Omega}$ for $\mathfrak{Y}=\mathfrak{B} \cap \mathfrak{X}$. 
From this we obtain

Lemma 3. If $\mathfrak{J}$ is an exceptional finite-dimensional simple Jordan algebra over a field $\Phi$ then for any Galois extension $\Omega$ of $\Phi$ the algebra $\mathfrak{\Im}_{\Omega}$ is semisimple, and either

(i) an exceptional division algebra;

(ii) a reduced exceptional algebra $\mathfrak{s}_{(}\left(\mathfrak{S}_{3}, \gamma\right)$;

(iii) a direct sum of two or more exceptional simple summands of type (i) or (ii).

Proof. Applying Lemma 2 to $\mathfrak{V}=\operatorname{Rad} \mathfrak{\Im}_{\Omega}$ (which is invariant under all ring automorphisms, hence under $G$ ) we see $\mathfrak{P}=\mathfrak{Y}_{\Omega}$ for $\mathfrak{Y}=\mathfrak{\Im} \cap \operatorname{Rad} \mathfrak{\Im}_{\Omega}$; by [9, p. 678] $\operatorname{Rad} \mathfrak{J}_{\Omega}$ is nil in the finite-dimensional case, so $\mathfrak{\Im} \cap \operatorname{Rad} \mathfrak{\Im}_{\Omega}$ is a nil ideal in $\mathfrak{\Im}$ which thus cannot contain 1 and hence by simplicity must be zero. From $\mathfrak{Y}=0$ we see $\mathfrak{B}=\operatorname{Rad} \Im_{\Omega}=0$ and $\mathfrak{\Im}_{\Omega}$ is semisimple. It is also still purely exceptional. By the structure theory $\left[4\right.$, p. 1079], [9, p. 678] if $\Im_{\Omega}$ is simple and exceptional it is of types (i) or (ii) above, while if it is not simple all its simple summands are exceptional (any direct summand of a purely exceptional algebra is purely exceptional).

Lemma 4. If $\mathfrak{\Im}$ is an exceptional finite-dimensional Jordan division algebra over a field $\Phi$ then there is a Galois extension $\Omega$ of $\Phi$ such that $\Im_{\Omega}$ is no longer a division algebra.

Proof. Suppose on the contrary that $\Im_{\Omega}$ is a division algebra for all Galois $\Omega$. Note that the same holds for $\mathfrak{\Im}_{\Omega}$ over $\Omega$ since $\left(\Im_{\Omega}\right)_{\mathrm{P}}=\mathfrak{\Im}_{\mathrm{P}}$ with $\mathrm{P} / \Phi$ Galois if $\mathrm{P} / \Omega$ and $\Omega / \Phi$ are. In particular, replacing $\Phi$ by some suitable $\Omega$ and $\Im$ by $\Im_{\Omega}$ if necessary we can assume the base field contains more than three elements. If some $x \in \mathfrak{J}$ satisfied a separable polynomial over $\Phi$ we could take $\Omega$ to be a splitting field of this polynomial; then there would be elements in $\Omega[x] \subset \Im_{\Omega}$ which are no longer invertible. This is impossible, so no element can satisfy a separable polynomial, and every element must satisfy a purely inseparable polynomial.

Consider the algebraic closure $\Omega$ of $\Phi . \operatorname{Rad} \Im_{\Omega}$ is nil and $\Im_{\Omega}$ is unital, so $\Im_{\Omega} / \operatorname{Rad} \Im_{\Omega}$ is a nonzero finite-dimensional semisimple purely exceptional Jordan algebra over the algebraically closed field $\Omega$; by the structure theory it is a direct sum of one or more split exceptional algebras $\mathfrak{S}\left(\mathfrak{C}_{3}\right)$. All we need to know is that $\mathfrak{\Im}_{\Omega}$ has a homomorphic image $\overline{\mathfrak{I}}_{\Omega}=\mathfrak{S}\left(\mathfrak{C}_{3}\right)$. Since any $x \in \mathfrak{I}$ is purely inseparable, $x^{p^{e}}=\alpha 1$ for $\alpha \in \Phi, x=\beta 1+z$ for $\beta \in \Omega$ and $z$ nilpotent in $\mathfrak{\Im}_{\Omega}$. Then $x^{k}=\beta^{k} 1+z_{k}$ with nilpotent $z_{k}$ for any $k$, and this remains true in $\bar{\Im}_{\Omega}: \bar{x}^{k}=\beta^{k} \overline{1}+\bar{z}_{k}$ for $\bar{z}_{k}$ nilpotent. Now in $\mathfrak{S}\left(\mathfrak{C}_{3}\right)$ the trace of any nilpotent element is zero and the trace of $\overline{1}$ is 3 , so $t(\alpha \overline{1}+\bar{w})$ $=3 \alpha$. From this we get the relations

$$
t(\bar{x})^{2}=3 t\left(\bar{x}^{2}\right), \quad t(\bar{x})^{3}=9 t\left(\bar{x}^{3}\right)
$$

for all $\bar{x} \in \overline{\mathfrak{J}}$. By our hypothesis that $\Phi$ contains more than three elements, these relations remain valid for all $\bar{y} \in \overline{\mathfrak{\Im}}_{\Omega}$ (note $\overline{\mathfrak{\Im}}$ spans $\bar{\Im}_{\Omega}$ over $\Omega$ ). Setting $\bar{y}=e_{1}=\bar{y}^{2}$ in $\mathfrak{S g}_{\mathrm{g}}\left(\mathfrak{S}_{3}\right)$ we see $1=t(\bar{y})^{2}=3 t\left(\bar{y}^{2}\right)=3$, so the characteristic is two. Then $t\left(\bar{y}^{3}\right)$ $=t(\bar{y})^{3}=t(\bar{y})^{2} t(\bar{y})=t\left(\bar{y}^{2}\right) t(\bar{y})$, so setting $\bar{y}=e_{1}+\omega e_{2}$ for $\omega \in \Omega$ we see $1+\omega^{3}$ 
$=\left(1+\omega^{2}\right)(1+\omega)$ so that $\omega^{2}+\omega=0$ for all $\omega$ in the algebraically closed field $\Omega$, which is ridiculous.

Lemma 5. If $\mathfrak{\Im}$ is an exceptional finite-dimensional simple Jordan algebra then $\Gamma(\mathfrak{\Im})=\Gamma_{0}(\mathfrak{\Im})$ and $\mathfrak{\Im}$ is outer-simple when considered as a ring.

Proof. If not, choose a $\mathfrak{\Im}$ of smallest possible dimension over its center $\Phi$ such that $\Gamma(\Im) \neq \Gamma_{0}(\mathfrak{\Im})$ (respectively such that $\mathfrak{\Im}$ contains a proper outer ideal $\mathfrak{R}$, i.e. an outer ideal which is not an ideal). By Proposition 5 (resp. by the fact that $\mathfrak{\Omega}_{\Omega}$ is outer but not an ideal in $\mathfrak{\Im}_{\Omega}$ ) the same is true of any $\mathfrak{\Im}_{\Omega}$. By Lemma 4 we can choose a Galois $\Omega$ so that $\Im_{\Omega}$ is not a division algebra. Also, $\Im_{\Omega}$ is not reduced since $\Gamma_{0}=\Gamma$ for $\mathfrak{S}_{\mathrm{C}}\left(\mathfrak{S}_{3}, \gamma\right)$ by Proposition 6 (resp. since $\mathfrak{S}_{\mathrm{C}}\left(\mathfrak{C}_{3}, \gamma\right)$ contains no proper outer ideals by Proposition 7). Thus by Lemma 3 (iii) $\Im_{\Omega}$ must be a direct sum of reduced exceptional algebras or exceptional division algebras of lower dimension over $\Omega$ (and hence even lower over their centers) than $\mathfrak{\Im}$ over $\Phi$. By minimality of $\mathfrak{\Im}$ these division algebras $\mathfrak{\Im}_{i}$ have $\Gamma\left(\mathfrak{\Im}_{i}\right)=\Gamma_{0}\left(\mathfrak{\Im}_{i}\right)$ (resp. all outer ideals in $\mathfrak{\Im}_{i}$ are ideals), as do the reduced algebras again by Proposition 6 (resp. Proposition 7), so by Proposition 4 (resp. Proposition 8$) \Gamma\left(\Im_{\Omega}\right)=\Gamma_{0}\left(\Im_{\Omega}\right)$ (resp. all outer ideals of $\Im_{\Omega}$ are ideals), which is a contradiction.

Putting all the lemmas together,

THEOREM 5. If $\mathfrak{\Im}$ is any exceptional finite-dimensional central simple Jordan algebra over $\Phi$ then $\mathfrak{\Im}$ is outer-simple and outer-central, so any extension $\mathfrak{\Im}_{\Omega}$ remains central simple.

Proof. This follows from Lemma 5 and Theorem 4 (recalling (24) and (25)).

THEOREM 6. Any exceptional finite-dimensional central simple Jordan algebra is a form of $\mathfrak{S}\left(\mathfrak{F}_{3}\right)$.

Proof. We apply Theorem 5 with $\Omega$ the algebraic closure; as noted before, by the structure theory $\mathfrak{I}_{\Omega}$ must be a reduced exceptional algebra $\mathfrak{S}_{\mathfrak{e}}\left(\mathfrak{C}_{3}, \gamma\right)$; over the algebraic closure $\mathfrak{C}$ is split, so we can take $\gamma=1$.

We can adopt a different approach. Rather than proving that certain Jordan algebras remain central simple under all extensions, we can show that all Jordan algebras remain central simple under certain extensions.

THEOREM 7. If $\mathfrak{\Im}$ is a finite-dimensional central simple Jordan algebra (not necessarily exceptional) over $\Phi$ then $\mathfrak{\Im}$ is central simple over $\Omega$ for any Galois extension $\Omega$ of $\Phi$.

This is enough to obtain Theorem 6: by Lemma 4 we can choose $\Omega$ so that $\Im_{\Omega}$ is not a division algebra, and $\mathfrak{\Im}_{\Omega}$ is still simple by Theorem 7 , so by Lemma $3 \Im_{\Omega}$ is a reduced $\mathfrak{S}_{\mathfrak{C}}\left(\mathfrak{C}_{3}, \gamma\right)$. Then $\mathfrak{\Im}$ is a form of $\mathfrak{S}_{\mathfrak{C}}\left(\mathfrak{C}_{3}\right)$.

Proof of Theorem 7. By Lemma $3 \mathfrak{\Im}_{\Omega}$ is still semisimple,

$$
\Im_{\Omega}=\bigoplus_{i=0}^{m} \Re_{i}
$$


where the $\Omega_{i}$ are simple unital $\Omega$-ideals. Each $g$ in the Galois group $G=G(\Omega / \Phi)$ induces a $\Phi$-linear automorphism of $\Im_{\Omega}$ by $(\omega \otimes x)^{g}=\omega^{g} \otimes x$ such that $\Im$ is precisely the set of fixed points of $G$. Thus each conjugate $\mathscr{\pi}_{i}^{g}(g \in G)$ is an $\Omega$-ideal which is $\Phi$-isomorphic to $\mathfrak{R}_{i}$, hence in particular is simple. But the only simple ideals in $\Im_{\Omega}$ are the $\Re_{i}$ since any ideal $\mathscr{N}$ is a direct sum of certain $\mathfrak{R}_{i}$ : if $e_{i}$ is the unit for $\mathfrak{\Re}_{i}$ then $\mathfrak{R}=U_{1} \mathfrak{R}=U_{e_{1}} \mathfrak{R} \oplus \cdots \oplus U_{e_{m}} \mathfrak{R}$ where $U_{e_{i}} \mathfrak{R}=\mathfrak{R} \cap \mathfrak{\Re}_{i}$ is either $\mathfrak{R}_{i}$ or 0 . Thus $g$ permutes the ideals $\mathfrak{\Re}_{i}: \mathfrak{K}_{i}^{g}=\mathfrak{\Re}_{g(i)}$. These permutations act transitively: $\mathfrak{\Omega}=\sum_{g \in G} \mathfrak{R}_{0}^{g}$ is a nonzero ideal in $\Re_{\Omega}$ which is clearly $G$-invariant, hence by Lemma

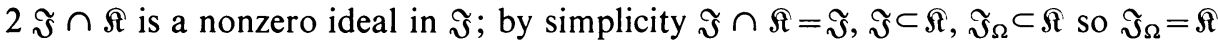
$=\sum \mathfrak{\Re}_{0}^{g}$ and every $\mathfrak{\Re}_{i}$ appears as a conjugate $\mathfrak{I}_{0}^{g}$ for some $g_{i}$. If we let

$$
H=\left\{h \in G \mid \mathfrak{\Re}_{0}^{h}=\mathfrak{R}_{0}\right\}
$$

then there is a $1-1$ correspondence between cosets $H g$ and the ideals $\Re_{i}$ given by $H g \leftrightarrow \Re_{0}^{g}$.

Consider the centroid $\Omega_{0} \supset \Omega$ of $\mathscr{N}_{0}$ (as a ring); by Theorem 3 this is a field. Since $H$ acts (by restriction) as a group of $\Phi$-automorphisms on $\Re_{0}$ it also acts as a finite group of automorphisms on the centroid by Proposition 3, so let $\Phi_{0} \supset \Phi$ be the fixed field of $H$. Since $g_{i}$ is a $\Phi$-isomorphism of $\Re_{0}$ onto $\Re_{i}=\Re_{0}^{g_{i}}$ it induces a $\Phi$ isomorphism of the centroid $\Omega_{0}$ of $\Omega_{0}$ onto the centroid $\Omega_{i}$ of $\Re_{i}$. For $T_{0} \in \Phi_{0}$ set $T=\bigoplus_{i=0}^{m} T_{0}^{g_{i}} \in \bigoplus \Gamma\left(\Re_{i}\right)=\Gamma\left(\Im_{\Omega}\right)$ by $\left(^{*}\right)$ and Proposition 4 . We claim $T^{g}=T$ for all $g \in G$, i.e. $T \circ g=g \circ T$ : it is enough to verify this on $x_{i}=x_{0}^{g_{i}}$ in $\Re_{i}=\mathfrak{\Re}_{0}^{g_{i}}$, and $T\left(x_{i}^{g}\right)=T\left(x_{0}^{g_{i} g}\right)$ $=T_{0}^{g}{ }^{g} X_{0}^{g} g^{g}$ (since $T=T_{0}^{g}$ on $\mathfrak{\Omega}_{0}^{g}$, noting that $T_{0}^{h}=T_{0}$ for $h$ in $H$ by definition of $\Phi_{0}$ ) $=\left(T_{0} x_{0}\right)^{g_{i} g}=\left(T_{0}^{g_{i}} x_{0}^{g}\right)^{g}=\left(T x_{i}\right)^{g}$. But this implies $T(\mathfrak{J}) \subset \mathfrak{\Im}: x$ belongs to $\mathfrak{\Im}$ if and only if $x^{g}=x$ for all $g$, in which case $(T x)^{g}=T^{g} x^{g}=T x$ implies $T x$ belongs to $\mathfrak{J}$ too. By Proposition 5, $T \in \Gamma\left(\Im_{\Omega}\right) \cap \operatorname{End}_{\Phi}(\Im)=\Gamma(\Im)$. But we are assuming that $\mathfrak{\Im}$ is central over $\Phi$, so $T=\alpha I$ for some $\alpha \in \Phi$. Hence its restriction $T_{0}$ to $\mathfrak{R}_{0}$ is also $\alpha I, T_{0}=\alpha I$ $\in \Phi I$ for all $T_{0}$ in $\Phi_{0}$. This shows $\Phi_{0}=\Phi$. Thus $H$ acts (perhaps not faithfully) as a finite group $\bar{H}$ of automorphisms of the field $\Omega_{0}$ having fixed field $\Phi$. By Galois theory [11, p. 29, Theorem 5] this implies $\Phi$ is of finite codimension in $\Omega_{0}$ and $\left[\Omega_{0}: \Phi\right]=[\bar{H}: 1]$. But then

$$
[G: 1]=[\Omega: \Phi] \leqq\left[\Omega_{0}: \Phi\right]=[\bar{H}: 1] \leqq[H: 1] \leqq[G: 1]
$$

implies $H=G$ and $\Omega_{0}=\Omega$ so that $\Im_{\Omega}=\Omega_{0}$ is simple with center $\Omega$.

6. Forms of $\mathfrak{H}\left(\mathfrak{C}_{3}\right)$. Our goal is to show that the two Tits Constructions yield precisely all exceptional finite-dimensional central-simple Jordan algebras. By Theorem 6 we may restrict our attention to forms of $\mathfrak{S}_{2}\left(\mathfrak{E}_{3}\right)$. Let us first note some properties of $\mathfrak{S}\left(\mathfrak{C}_{3}\right)$ which are inherited by any of its forms $\mathfrak{\Im}$ :

(i) $\mathfrak{I}$ contains no nonzero absolute zero divisors;

(ii) $\mathfrak{\Im}$ is exceptional and central simple;

(iii) $\mathfrak{I}$ is generically algebraic of degree 3 and 27 -dimensional over its center.

Also, as we have seen many times, $\Im$ is either a Jordan division algebra or a reduced exceptional algebra $\mathfrak{S}_{\mathfrak{c}}\left(\mathfrak{S}_{3}, \gamma\right)$. 
The basic fact that makes the constructions work is

LEMma 6. Any form $\mathfrak{\Im}$ of the split exceptional algebra contains a subalgebra $\mathfrak{\Re}$ of the form $\mathfrak{A}^{+}$for $\mathfrak{A}$ a central simple associative algebra of degree 3 over $\Phi$, or of the form $\mathfrak{S}\left(\mathfrak{A},{ }^{*}\right)$ for $\mathfrak{A}$ central simple of degree 3 over a quadratic extension $\Omega$ of $\Phi$, with involution * of second kind having $\Phi$ as fixed field.

Proof. We first want to get rid of the case where $\Phi$ is finite. In this case the cubic norm form $N(x)$ in 27 variables has a nontrivial zero by a theorem of ArtinChevalley: $N(x)=0$ for $x \neq 0$. Since $\mathfrak{I}$ is generically algebraic this means $x$ is not invertible (see (5)), and $\mathfrak{I}$ is not a division algebra. Hence $\mathfrak{I}$ is reduced.

We might as well settle the reduced case in general (whether $\Phi$ is finite or not). If $\mathfrak{\Im}=\mathfrak{S}\left(\mathfrak{C}_{3}, \gamma\right)$ let $\mathfrak{D}$ be a 2-dimensional composition subalgebra of the Cayley algebra $\mathfrak{C}$. If $\mathfrak{D}$ is split, $\mathfrak{D}=\Phi f_{1}+\Phi f_{2}$ for $f_{1}, f_{2}$ orthogonal idempotents with $f_{1}^{*}=f_{2}$, then $\mathfrak{C}$ is split and we could assume $\gamma=1, \mathfrak{J}=\mathfrak{S}\left(\mathfrak{C}_{3}\right)$. In this case $\mathfrak{\Im}$ contains a subalgebra $\mathfrak{I}$ isomorphic to $\mathfrak{S}\left(\mathfrak{D}_{3}\right)=\mathfrak{S}\left((\Phi \oplus \Phi)_{3}\right)$. Now the map

$$
\left(\alpha_{i j}\right) \stackrel{\varphi}{\longrightarrow} \sum_{i=1}^{3} \alpha_{i i} e_{i i}+\sum_{i=1}^{3}\left(\alpha_{j k} f_{1}+\alpha_{k j} f_{2}\right)\left(e_{j k}+e_{k j}\right)
$$

((ijk) a cyclic permutation of (123)) is an isomorphism of $\Phi_{3}^{+}$onto $\mathfrak{S}\left((\Phi \oplus \Phi)_{3}\right)$. Using Theorem 1 and the fact that a nondegenerate norm determines the adjoint as in (12) (ii), it is enough if $\varphi(1)=\tilde{l}$ and $\tilde{N}(\varphi(x))=N(x)$; the first is clear, the second follows from $\tilde{N}(\varphi(x))=\alpha_{11} \alpha_{22} \alpha_{33}-\sum_{i} \alpha_{i i}\left(\alpha_{j k} \alpha_{k j}\right)+\left(\alpha_{23} \alpha_{31} \alpha_{12}+\alpha_{32} \alpha_{13} \alpha_{21}\right)=\operatorname{det}\left(\alpha_{i j}\right)$ $=N(x)$ by [3, p. 502]. Thus $\mathfrak{N} \cong \mathfrak{A}^{+}$for $\mathfrak{A}=\Phi_{3}$. In case $\mathfrak{D}$ is not split it is a quadratic field $\Omega$ with involution of second kind, so $\mathfrak{\Im}$ contains $\mathfrak{R} \cong \mathfrak{S}\left(\mathfrak{D}_{3}, \gamma\right)=\mathfrak{S}\left(\Omega_{3}, \gamma\right)=$ $\mathfrak{S C}\left(\mathfrak{R},{ }^{*}\right)$ where $\mathfrak{A}=\Omega_{3}$ is central simple of degree 3 over $\Omega$ and the involution $x^{*}=\gamma^{-1} \bar{x}^{t} \gamma\left(\gamma=\operatorname{diag}\left\{\gamma_{1}, \gamma_{2}, \gamma_{3}\right\}\right.$ for $\left.\gamma_{i} \in \Phi\right)$ is of second kind on $\mathfrak{A}$ with fixed field $\Phi$.

So now assume $\Phi$ is infinite. We set out to find elements $x, y$ in $\Im$ which generate a subalgebra $\mathfrak{N}=\Phi[x, y]$ of the desired form. If the discriminant $\delta(x)$ (see $\S 1$ ) vanished identically on $\mathfrak{\Im}$ it would vanish identically on any $\mathfrak{\Im}_{\Omega}$ since $\Phi$ is infinite, which it does not (some $\mathfrak{J}_{\Omega} \cong \mathfrak{S}_{(}\left(\mathfrak{夭}_{3}\right)$ ), so we can fix an $x$ in $\mathfrak{\Im}$ with $\delta(x) \neq 0$. Such an $x$ has distinct characteristic roots, so in some $\Im_{\Omega}$ we have $x=\alpha_{1} e_{1}+\alpha_{2} e_{2}+\alpha_{3} e_{3}$ for $\alpha_{i} \in \Omega, e_{i}$ nonzero orthogonal idempotents in $\Omega[x]$. We can use the $e_{i}$ to coordinatize $\mathfrak{\Im}_{\Omega}$, so $\mathfrak{J}_{\Omega} \cong \mathfrak{S}_{(}\left(\mathfrak{C}_{3}\right)$ where the $e_{i}$ correspond to the diagonal idempotents $e_{i i}$. Then any $y$ in $\mathfrak{\Im}\left(\right.$ or $\mathfrak{I}_{\Omega}$ ) has the form

$$
y=\sum \beta_{i} e_{i}+\sum b_{i}[j k] .
$$

We have a polynomial map $\Im \rightarrow \Omega$ by $F(y)=n\left(b_{1}\right) n\left(b_{2}\right) n\left(b_{3}\right)$ where $n$ is the norm in $\mathfrak{E}$. $F$ cannot vanish on all $y \in \mathfrak{\Im}$ since then its extension to $\Im_{\Omega}$ would vanish identically, which it does not (take $y$ with $b_{1}=b_{2}=b_{3}=1$ ). Thus for $y$ in the dense set $F(y) \neq 0$ the $b_{i}$ in $\left(^{*}\right)$ are all invertible. We can use $b_{3}[12]$ and $b_{2}[31]$ to recoordinatize $\mathfrak{J}_{\Omega}$ as $\mathfrak{S}_{(}\left(\mathfrak{E}_{3}, \gamma\right)$ so that $x$ still has the form $x=\sum \alpha_{i} e_{i}$ but $y$ looks like

$$
y=\sum \beta_{i} e_{i}+1[12]+1[31]+b[23]
$$


for some $b \in \mathfrak{C}$. In this case $\Omega[x, y] \cong \mathfrak{S}\left(\mathfrak{D}_{3}, \gamma\right)$ for $\mathfrak{D}=\Omega 1+\Omega b$. Suppose $\mathfrak{D}$ is not a 2-dimensional composition subalgebra of $\mathfrak{c}$. Then some $z=\alpha 1+\beta b$ has $n(z)$ $=t(1, z)=t\left(b^{*}, z\right)=0$, where $\alpha$ and $\beta$ are not both zero (though $z$ may be zero). We cannot have $\beta=0$, or else $z=\alpha 1$ for $\alpha \neq 0$ would have $n(z)=\alpha^{2} \neq 0$. So $\beta \neq 0$, and $t(1, z)=0 \Rightarrow t(b)=-2 \alpha \beta^{-1} ;$ similarly $t\left(b^{*}, z\right)=0 \Rightarrow 2 n(b)=-\alpha \beta^{-1} t(b) \Rightarrow 4 n(b)$ $=t(b)^{2}$. Thus if $\mathcal{D}$ is not of dimension 2 or is degenerate we have $G(y)=4 n(b)$ $-t(b)^{2}=0$.

Now $G$ is a polynomial function of $y$ (even though the coordinatization $\left({ }^{* *}\right)$ in which $b$ appears changes along with $y$ ), since in terms of the original fixed coordinatization $\left(^{*}\right)$ we have $n(b)=n\left(b_{1}\right) n\left(b_{2}\right)^{-1} n\left(b_{3}\right)^{-1}$ and $t(b)=$ $t\left(b_{1} b_{2} b_{3}\right) n\left(b_{2}\right)^{-1} n\left(b_{3}\right)^{-1}$. If we choose $y$ in $\Im$ so that $F(y) \neq 0$ and $G(y) \neq 0$ then $\mathfrak{R}=\Phi[x, y]$ has $\mathfrak{\Omega}_{\Omega}=\Omega[x, y] \cong \mathfrak{S}_{(}\left(\mathfrak{D}_{3}, \gamma\right)$ for $\mathfrak{D}$ a 2 -dimensional composition subalgebra of $\mathfrak{C}$. By assuming $\Omega$ is algebraically closed we get $\mathscr{D}$ and $\subseteq$ split. Hence by the calculations for the reduced case, $\mathfrak{R}_{\Omega}=\Omega_{3}^{+}$. Thus $\mathfrak{R}_{\Omega}=\mathfrak{T}\left(\operatorname{su}\left(\Re_{\Omega}\right), \pi\right)$ and hence $\mathfrak{K}=$ $\mathfrak{S}(\operatorname{su}(\Re), \pi)$ by [2, pp. 208-210] where su denotes the special universal envelope with main involution $\pi$. Since su $(\Re)_{\Omega}=\operatorname{su}\left(\Omega_{\Omega}\right)=\Omega_{3} \oplus \Omega_{3}^{0}$ with center $\Omega \oplus \Omega$ a quadratic extension of the fixed field $\Omega$ of $\pi$, su $(\Re)$ is either a central simple algebra $\mathfrak{A}$ of degree 3 with center a quadratic extension of the fixed field $\Phi$ of $\pi$ (so $\pi$ is of second kind), or else it is a direct sum $\mathfrak{A} \oplus \mathfrak{A}^{0}$ of a central simple algebra $\mathfrak{A}$ of degree 3 over $\Phi$ with its opposite algebra $\mathfrak{A}^{0}$ (where $\pi$ is the exchange involution). Then $\mathfrak{R}=\mathfrak{S}\left(\mathfrak{A},{ }^{*}\right)$ or $\mathfrak{H}^{+}$as asserted.

THEOREM 8. If $\Im$ is a form of $\mathfrak{S}_{\mathfrak{E}}\left(\mathfrak{C}_{3}\right)$ which contains a subalgebra of the form $\mathfrak{A}^{+}$ for $\mathfrak{A}$ central simple of degree 3 over $\Phi$, then $\mathfrak{\Im} \cong(\mathfrak{A}, \mu)$ for some $\mu \in \Phi$.

Proof. The motivation for the proof is roughly as follows. Recall [3, p. 507] that $\mathfrak{I}(\mathfrak{A}, \mu)$ is given by

$$
\mathfrak{I}(\mathfrak{A}, \mu)=\mathfrak{A}_{0} \oplus \mathfrak{A}_{1} \oplus \mathfrak{A}_{2}
$$

where the $\mathfrak{A}_{i}$ are copies of $\mathfrak{A}$, and where the norm $N$, adjoint \#, and trace $T$ of $\mathfrak{\Im}(\mathfrak{A}, \mu)$ are

$$
\begin{aligned}
N(a, b, c) & =n(a)+\mu n(b)+\mu^{-1} n(c)-t(a b c), \\
(a, b, c)^{\#} & =\left(a^{\#}-b c, \mu^{-1} c^{\#}-a b, \mu b^{\#}-c a\right), \\
T\left((a, b, c),\left(a^{\prime}, b^{\prime}, c^{\prime}\right)\right) & =t\left(a, a^{\prime}\right)+t\left(b, c^{\prime}\right)+t\left(c, b^{\prime}\right)
\end{aligned}
$$

for an element $(a, b, c)$ of the direct sum, in terms of the norm $n$, adjoint \#, and trace $t$ on $\mathfrak{A}$. The given subalgebra $\mathfrak{A}^{+}$will be our $\mathfrak{A}_{0}$, and we must find subspaces of our $\mathfrak{\Im}$ that act like $\mathfrak{A}_{1}$ and $\mathfrak{A}_{2}$. The idea is that $\mathfrak{A}_{1}\left(\right.$ or $\left.\mathfrak{A}_{2}\right)$ is determined precisely as the subspace of the orthogonal complement $\mathfrak{A}_{0}^{\perp}$ of $\mathfrak{A}_{0}$ under $T$ which acts like a left (or right) $\mathfrak{A}_{0}$-module under the composition $a \cdot m=-a \times m$ (or $m \cdot a=-a \times m$ ).

Now for the proof. Since $\mathfrak{J}_{\Omega} \cong \mathfrak{S}\left(\mathfrak{C}_{3}\right)$ is generically algebraic with nondegenerate trace form, the same must be true of $\mathfrak{\Im}$ itself. Given a subalgebra of the form $\mathfrak{A}^{+}$, 
let $\mathfrak{M}=\mathfrak{A}^{\perp}$ be the orthogonal complement of $\mathfrak{A}$ relative to $T(x, y)$ (we regard $\mathfrak{A}$ as imbedded in $\mathfrak{\Im}$ in such a way that the operations in $\mathfrak{A}^{+}$are induced by those in $\mathfrak{\Im}$; hence the generic norm and trace in $\mathfrak{A}$ are those induced by $\mathfrak{F}$, consequently $T(x, y)$ is nondegenerate on $\mathfrak{A})$. We note that $\mathfrak{A} \times \mathfrak{M} \subset \mathfrak{M}=\mathfrak{U}^{\perp}$ since $T(\mathfrak{A} \times \mathfrak{M}, \mathfrak{A})$ $=T(\mathfrak{M}, \mathfrak{A} \times \mathfrak{A})($ by $(21)) \subset T(\mathfrak{M}, \mathfrak{A} \#) \subset T(\mathfrak{M}, \mathfrak{A})=0$. Thus

$$
S_{a}: m \rightarrow-a \times m
$$

defines an endomorphism of $\mathfrak{M}$ for each $a$ in $\mathfrak{A}$. We claim

$$
S: a \rightarrow S_{a}
$$

is a specialization of $\mathfrak{A}$ in End $(\mathfrak{M})$. The condition

$$
S(c)=I
$$

follows from $S_{c} m=-c \times m=-T(m) c+m=m$ because $T(\mathfrak{M})=T(\mathfrak{M}, c)=0$, and

follows from

$$
S(a b a)=S(a) S(b) S(a)
$$

$$
\begin{aligned}
S_{a b a} m & \left.=-(a b a) \times m=-\left\{T(a, b) a-a^{\#} \times b\right\} \times m \quad \text { (because of Theorem } 1 \text { for } \mathfrak{A}^{+}\right) \\
& =-T(a, b) a \times m+m \times\left(a^{\#} \times b\right) \\
& =-T(a, b) a \times m-a \times(\{a \times m\} \times b)+\left(\left.\partial_{m} N\right|_{a}\right) b+T(a, b) a \times m+T(m, b) a^{\#} \\
& =-a \times(b \times(a \times m))+T(m, b) a^{\#}+T\left(a^{\#}, m\right) b \quad(\text { by }(12)(\text { ii })) \\
& =-a \times(b \times(a \times m)) \quad\left(\text { since } m \text { is orthogonal to } a^{\#}, b \in \mathfrak{A}\right)=S_{a} S_{b} S_{a} m .
\end{aligned}
$$

The special universal envelope of $\mathfrak{A}^{+}$is su $\left(\mathfrak{A}^{+}\right)=\mathfrak{A} \oplus \mathfrak{A}^{0}$ as noted before (the argument in [1, p. 210] extends to quadratic Jordan algebras, by remarks in $\left[6\right.$, p. 1333]). Thus the specialization $S: \mathfrak{A}^{+} \rightarrow$ End $(\mathfrak{M})$ extends uniquely to a homomorphism (again denoted by $S$ ) of associative algebras $\mathfrak{A} \oplus \mathfrak{A}^{0} \rightarrow$ End $(\mathfrak{M})$. But then $S=S_{1} \oplus S_{2}$ where $S\left(a \oplus b^{0}\right)=S_{1}(a)+S_{2}(b)$; here $S_{1}, S_{2}: \mathfrak{A} \rightarrow$ End $(\mathfrak{M})$ are homomorphisms and antihomomorphisms respectively satisfying $S_{1}(a) S_{2}(b)$ $=S_{2}(b) S_{1}(a)=0$ for all $a, b \in \mathfrak{A}$. We get $I=S(c)=S_{1}(c)+S_{2}(c)=E_{1}+E_{2}$ where the $E_{i}=S_{i}(c)$ are supplementary orthogonal idempotent operators. This leads to a decomposition

(31) $\mathfrak{M}=\mathfrak{M}_{1} \oplus \mathfrak{M}_{2}$ for $\mathfrak{M}_{i}=E_{i}(\mathfrak{M})$. Since $S_{i}(a)=0$ on $\mathfrak{M}_{j}=S_{j}(c) \mathfrak{M}(i \neq j)$ we may regard $S_{i}$ as a homomorphism or antihomomorphism of $\mathfrak{A}$ into End $\left(\mathfrak{M}_{i}\right)$. This turns $\mathfrak{M}_{1}$ into a left and $\mathfrak{M}_{2}$ into a right $\mathfrak{A}$-module by $a \cdot m_{1}=-a \times m_{1}, m_{2} \cdot a=-m_{2} \times a$.

This implies $a \times(b \times m)$ equals $-(a b) \times m$ on $\mathfrak{M}_{1}$ and $-(b a) \times m$ on $\mathfrak{M}_{2}$. This intrinsically characterizes $\mathfrak{M}_{1}$ and $\mathfrak{M}_{2}$,

$$
\begin{aligned}
& \mathfrak{M}_{1}=\{m \in \mathfrak{M} \mid a \times(b \times m)=-(a b) \times m \text { for all } a, b \in \mathfrak{A}\}, \\
& \mathfrak{M}_{2}=\{m \in \mathfrak{M} \mid a \times(b \times m)=-(b a) \times m \text { for all } a, b \in \mathfrak{A}\} .
\end{aligned}
$$


To see this, let $\mathfrak{M}_{i}^{\prime}$ denote the right hand sides of (32). Then $\mathfrak{M}_{i}^{\prime}$ is a subspace of $\mathfrak{M}$ containing $\mathfrak{M}_{i}$, and if we can show $\mathfrak{M}_{1}^{\prime} \cap \mathfrak{M}_{2}^{\prime}=0$ then $\mathfrak{M}=\mathfrak{M}_{1}^{\prime} \oplus \mathfrak{M}_{2}^{\prime}$ and $\mathfrak{M}_{i}^{\prime}=\mathfrak{M}_{i}$ by (31). But if $m \in \mathfrak{M}_{1}^{\prime} \cap \mathfrak{M}_{2}^{\prime}$ then $(a b) \times m=(b a) \times m$ for all $a, b$, so $[a, b]=a b-b a$ belongs to Ann $(m)=\{a \in \mathfrak{A} \mid a \times m=0\}$. By (32) Ann $(m)$ is a 2-sided ideal in $\mathfrak{A}$ containing all commutators. Since $\mathfrak{A}$ is not commutative, this ideal is not 0 , and since $\mathfrak{A}$ is simple it must be all of $\mathfrak{A}$. But then $c \in$ Ann $(m)$ implies $m=-c \times m=0$.

Next we wish to establish

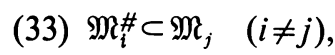

(34) $N\left(\mathfrak{M}_{i}\right) \neq 0$.

It would be desirable to have an intrinsic proof of this, but we will settle for the following field-extension argument. We claim it is enough to prove (33) and

(34)' $T\left(\mathfrak{M}_{i}, \mathfrak{M}_{i} \times \mathfrak{M}_{i}\right) \neq 0$

in some extension $\mathfrak{\Im}_{\Omega}$. For the $\mathfrak{M}_{i}\left(\mathfrak{H}_{\Omega}\right)$ determined by $\mathfrak{A}_{\Omega}$ in $\mathfrak{\Im}_{\Omega}$ are just $\mathfrak{M}_{i \Omega}$ because of the linearity of the criterion (32), so if $\mathfrak{M}_{i}\left(\mathfrak{A}_{\Omega}\right)^{\# \subset} \subset \mathfrak{M}_{j}\left(\mathfrak{A}_{\Omega}\right)$ we will have $\mathfrak{M}_{i}^{\#} \subset \mathfrak{M}_{j}$. Also, since (34)' is linear it will hold in $\mathfrak{\Im}$ if and only if it holds in $\mathfrak{\Im}_{\Omega}$. We claim that (34)' implies (34): if $N\left(\mathfrak{M}_{i}\right)=0$ then for any $m_{i}, m_{i}^{\prime}, m_{i}^{\prime \prime}$ in $\mathfrak{M}_{i}$ we would have $0=N\left(m_{i}+m_{i}^{\prime}+m_{i}^{\prime \prime}\right)-N\left(m_{i}+m_{i}^{\prime}\right)-N\left(m_{i}^{\prime}+m_{i}^{\prime \prime}\right)-N\left(m_{i}^{\prime \prime}+m_{i}\right)+N\left(m_{i}\right)+N\left(m_{i}^{\prime}\right)+N\left(m_{i}^{\prime \prime}\right)$ $=T\left(m_{i}, m_{i}^{\prime} \times m_{i}^{\prime \prime}\right)$ by (12.ii) and (21). Thus if (34) holds in $\Im_{\Omega}$ we will have (34) in $\Im$.

Let us then digress to examine the situation in $\mathfrak{\Im}_{\Omega}$. If $\mathfrak{\Im}$ contains $\mathfrak{\Re} \cong \mathfrak{A}^{+}$we may choose $\Omega$ algebraically closed so $\Im_{\Omega}$ contains $\mathfrak{\Omega}_{\Omega} \cong \mathfrak{A}_{\Omega}^{+} \cong \Omega_{3}^{+}$. We can use the $e_{i i}$ and $e_{i j}+e_{j i}$ of $\Omega_{3}$ to coordinatize both $\mathfrak{I}_{\Omega}$ and $\mathfrak{\Omega}_{\Omega}$ simultaneously, so $\mathfrak{I}_{\Omega} \cong \mathfrak{S}_{\mathfrak{g}}\left(\mathfrak{E}_{3}\right)$ and $\mathfrak{\Re}_{\Omega} \cong \mathfrak{S}\left(\mathfrak{D}_{3}\right)$ for $\mathfrak{D}=\Omega f_{1}+\Omega f_{2}$ a split subalgebra of the split Cayley algebra $\mathfrak{C}$. The $f_{i}$ are orthogonal idempotents with $f_{i}^{*}=f_{j}$; relative to them we have the Peirce decomposition $\mathfrak{E}=\mathfrak{C}_{11} \oplus \mathfrak{C}_{12} \oplus \mathfrak{C}_{21} \oplus \mathfrak{C}_{22}$ with $\mathfrak{E}_{i i}=\Omega f_{i}$. We can choose $x_{1}, x_{2}, x_{3}$ in $\mathfrak{E}$ so that the $f_{1} x_{s} f_{2}(s=1,2,3)$ are a basis for $\mathfrak{C}_{12}$ and the $f_{2} x_{s} f_{1}$ a basis for $\mathfrak{C}_{21}$. We know

$$
\begin{aligned}
\mathfrak{M}_{0}=\mathfrak{N}_{\Omega} & =\left\{\sum \alpha_{i} e_{i}+\sum a_{i}[j k] \mid a_{i} \in \mathfrak{S}_{11}+\mathfrak{C}_{22}\right\} \\
& =\left\{\sum \alpha_{i i} e_{i}+\sum\left(\alpha_{j k} f_{1}+\alpha_{k j} f_{2}\right)[j k]\right\}
\end{aligned}
$$

is $\mathfrak{S}\left(\mathfrak{D}_{3}\right)$ and isomorphic to $\Omega_{3}^{+}$under $m_{0} \rightarrow\left(\alpha_{i j}\right)$ by (26). Also

$$
\mathfrak{M}_{1}=\left\{\sum b_{i}[j k] \mid b_{i} \in \mathfrak{C}_{21}\right\}=\left\{\sum\left(\sum_{s} \beta_{i s} f_{2} x_{s} f_{1}\right)[j k]\right\}
$$

is linearly isomorphic to $\Omega_{3}$ under $m_{1} \rightarrow\left(\beta_{i j}\right)$. Similarly

$$
\mathfrak{M}_{2}=\left\{\sum c_{i}[j k] \mid c_{i} \in \mathfrak{C}_{12}\right\}=\left\{\sum\left(\sum_{s} \gamma_{i s} f_{1} x_{s} f_{2}\right)[j k]\right\}
$$

is isomorphic to $\Omega_{3}$ under $m_{2} \rightarrow\left(\gamma_{i j}\right)$. We claim the $\mathfrak{M}_{i}$ are the $\mathfrak{M}_{i}\left(\Omega_{3}\right)$ we are 
looking for. The operators in $\mathfrak{S}_{(}\left(\mathfrak{C}_{3}\right)$ are given by the Freudenthal Construction [3, p. 502], so for $x=\sum \alpha_{i} e_{i}+\sum a_{i}[j k]$

$$
\begin{aligned}
x^{\#} & =\sum\left\{\alpha_{j} \alpha_{k}-n\left(a_{i}\right)\right\} e_{i}+\sum\left\{\left(a_{j} a_{k}\right)^{*}-\alpha_{i} a_{i}\right\}[j k], \\
T(x, y) & =\sum \alpha_{i} \beta_{i}+\sum t\left(a_{i}^{*}, b_{i}\right) .
\end{aligned}
$$

Thus $-m_{0} \times m_{1}=\sum n\left(a_{i}, b_{i}\right) e_{i}+\sum\left\{\alpha_{i} b_{i}-\left(a_{j} b_{k}+b_{j} a_{k}\right)^{*}\right\}[j k]$. Now $n(a, b)=t\left(a^{*}, b\right)$ and $\mathfrak{C}_{11}+\mathfrak{C}_{22}$ is orthogonal to $\mathfrak{C}_{12}+\mathfrak{C}_{21}$ under $t$, so $n\left(a_{i}, b_{i}\right)=0$. Also $b^{*}=-b$ on $\mathfrak{C}_{12}+\mathfrak{C}_{21}$, so $-m_{0} \times m_{1}=\sum\left\{\alpha_{i} b_{\imath}+b_{k} a_{j}^{*}+a_{k}^{*} b_{j}\right\}[j k]$. If $m_{0} \rightarrow\left(\alpha_{i j}\right), m_{1} \rightarrow\left(\beta_{i j}\right)$ then

$$
\begin{aligned}
\alpha_{i} b_{i}+b_{k} a_{j}^{*}+a_{k}^{*} b_{j}= & \alpha_{i i}\left\{\sum \beta_{i s} f_{2} x_{s} f_{1}\right\}+\left\{\sum \beta_{k s} f_{2} x_{s} f_{1}\right\}\left(\alpha_{k i} f_{2}+\alpha_{i k} f_{1}\right) \\
& +\left(\alpha_{i j} f_{2}+\alpha_{j i} f_{1}\right)\left\{\sum \beta_{j s} f_{2} x_{s} f_{1}\right\} \\
= & \sum_{s}\left(\alpha_{i i} \beta_{i s}+\alpha_{i k} \beta_{k s}+\alpha_{i j} \beta_{j s}\right) f_{2} x_{s} f_{1}
\end{aligned}
$$

so that $-m_{0} \times m_{1} \rightarrow\left(\beta_{i j}^{\prime}\right)$ for $\beta_{i s}^{\prime}=\sum_{t} \alpha_{i t} \beta_{t s}$, i.e. $\left(\beta_{i j}^{\prime}\right)=\left(\alpha_{i j}\right)\left(\beta_{i j}\right)$. Similarly $-m_{2} \times m_{0}$ $\rightarrow\left(\gamma_{i j}\right)\left(\alpha_{i j}\right)$. This shows that $\mathfrak{M}_{1}, \mathfrak{M}_{2}$ are the spaces which act as left, right $\Omega_{3^{-}}$ modules. To verify (33), note that $m_{1}^{\#}=\sum\left(a_{j} a_{k}\right)^{*}[j k]$ belongs to $\mathfrak{M}_{2}$ since $n\left(\mathfrak{E}_{21}\right)=0$

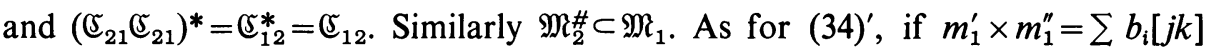
for $b_{i}=\left(a_{j}^{\prime} a_{k}^{\prime \prime}+a_{j}^{\prime \prime} a_{k}^{\prime}\right)^{*}$ then

$$
T\left(m_{1}, m_{1}^{\prime} \times m_{1}^{\prime \prime}\right)=\sum t\left(a_{i}^{*}, b_{i}\right)=\sum t\left(a_{i}^{*},\left(a_{j}^{\prime} a_{k}^{\prime \prime}+a_{j}^{\prime \prime} a_{k}^{\prime}\right)^{*}\right)=\sum t\left(a_{i}, a_{j}^{\prime} a_{k}^{\prime \prime}+a_{j}^{\prime \prime} a_{k}^{\prime}\right) .
$$

Since we can find $a_{1}, a_{2}^{\prime}, a_{3}^{\prime \prime}$ in $\mathfrak{\mho}_{21}$ with $t\left(a_{1}, a_{2}^{\prime} a_{3}^{\prime \prime}\right) \neq 0$ we have established (34)'.

Having established (33) and (34), choose $m_{1} \in \mathfrak{M}_{1}$ with $N\left(m_{1}\right)=\mu \neq 0$. Then $m_{2}=\mu^{-1} m_{1}^{\#}$ belongs to $\mathfrak{M}_{2}$ by (33) and has $N\left(m_{2}\right)=\mu^{-3} N\left(m_{1}^{\#}\right)=\mu^{-3} N\left(m_{1}\right)^{2}$ (by (22) (iii)) $=\mu^{-1}$. If $a \in \mathfrak{A}$ then $a \times m_{i}=0 \Rightarrow 0=m_{i}^{\#} \times\left(m_{i} \times a\right)=N\left(m_{i}\right) a+T\left(m_{i}^{\#}, a\right) m_{i}$ (by (22) (i)) $=N\left(m_{i}\right) a$ (since $m_{i}^{\#} \in \mathfrak{M}_{j}$ is orthogonal to $\mathfrak{U}$ ) $\Rightarrow a=0$. Thus the map $a \rightarrow$ $S_{a} m_{i}=-a \times m_{i}$ is injective. This gives us an isomorphism of $\mathfrak{A}$ with $\mathfrak{A}_{1}=\mathfrak{A} \cdot m_{1}$ as left $\mathfrak{A}$-module and with $\mathfrak{A}_{2}=m_{2} \cdot \mathfrak{A}$ as right $\mathfrak{A}$-module. Setting $\mathfrak{A}_{0}=\mathfrak{A}$ we get $\operatorname{dim}\left(\mathfrak{A}_{0} \oplus \mathfrak{A}_{1} \oplus \mathfrak{A}_{2}\right)=9+9+9=\operatorname{dim} \mathfrak{A}_{\Omega}=\operatorname{dim}\left(\mathfrak{U} \oplus \mathfrak{M}_{1} \oplus \mathfrak{M}_{2}\right)$ since $\mathfrak{I}$ is a form of $\mathfrak{S}\left(\mathfrak{C}_{3}\right)$. Thus

$$
\mathfrak{M}_{1}=\mathfrak{A}_{1}=\mathfrak{A} \cdot m_{1}, \quad \mathfrak{M}_{2}=\mathfrak{A}_{2}=m_{2} \cdot \mathfrak{A}, \quad \mathfrak{I}=\mathfrak{A}_{0} \oplus \mathfrak{A}_{1} \oplus \mathfrak{A}_{2} .
$$

If we let $c_{i}(i=0,1,2)$ denote the image of $a \in \mathfrak{A}$ in $\mathfrak{A}_{i}$ under the above isomorphisms we claim

$$
N\left(a_{0}\right)=n(a), \quad N\left(a_{1}\right)=\mu n(a), \quad N\left(a_{2}\right)=\mu^{-1} n(a) .
$$

The first follows since $\mathfrak{A}^{+}=\mathfrak{U}_{0}$ is imbedded in $\mathfrak{J}$, the others because $N\left(a \times m_{\mathfrak{i}}\right)$ $+N(a) N\left(m_{i}\right)=T\left(m_{i}^{\#}, a\right) T\left(m_{i}, a^{\#}\right)=0$ by (22) (iv), so $N\left(-a \times m_{i}\right)=N(a) N\left(m_{i}\right)$. Note by (33) that $T\left(\mathfrak{U}_{i}^{\#}\right) \subset T\left(\mathfrak{A}_{j}\right)=0$ for $i, j=1,2$ implies $T\left(\mathfrak{U}_{i} \times \mathfrak{A}_{i}\right)=0$; we have seen before that $T\left(\mathfrak{U}_{i}\right)=0$, so from (20)

(37) $T\left(\mathfrak{U}_{i}, \mathfrak{A}_{i}\right)=0$. 
Hence (33), (37), and orthogonality give

(38) $T\left(a_{0}^{\#}, b_{1}+c_{2}\right)=T\left(b_{1}^{\#}, c_{2}+a_{0}\right)=T\left(c_{2}^{\#}, a_{0}+b_{1}\right)=0$

for any $a, b, c \in \mathfrak{A}$. We claim further

(39) $T\left(a_{0}, b_{1} \times c_{2}\right)=-t(a b c)$.

We have

$$
\begin{aligned}
T\left(a_{0}, b_{1} \times c_{2}\right) & =T\left(a,\left(b \times m_{1}\right) \times\left(c \times m_{2}\right)\right)=\mu^{-1} T\left(a \times\left(b \times m_{1}\right), c \times m_{1}^{\#}\right) \\
& =-\mu^{-1} T\left((a b) \times m_{1}, c \times m_{1}^{\#}\right) \quad(\text { by }(32)) \\
& =-\mu^{-1} T\left(\left\{(a b) \times m_{1}\right\} \times m_{1}^{\#}, c\right) \\
& =-\mu^{-1} T\left(N\left(m_{1}\right) a b+T\left(m_{1}, a b\right) m_{1}^{\#}, c\right) \quad(\text { by }(22)(\mathrm{i})) \\
& =-T(a b, c) \quad\left(\text { since } N\left(m_{1}\right)=\mu \text { and } m_{1} \text { is orthogonal to } a b\right) \\
& =-t(a b c)
\end{aligned}
$$

since $T$ coincides with the associative generic trace form $t$ on $\mathfrak{A}$.

These formulas allow us to establish the theorem. By Theorem 1 we know $\mathfrak{\Im}=\mathfrak{\Im}(N, \#, c)$ for $N$ the generic norm, \# the adjoint, $c$ the unit; since $N$ is nondegenerate, \# is uniquely determined by $N$ from (12.ii), so [3, p. 504] $\mathfrak{\Im}=\Im(N, c)$. But $\Im(N, c)$ and $\Im(\mathfrak{A}, \mu)$ (for $\mu=N\left(m_{1}\right)$ as above) both have the same vector space structure $\mathfrak{A}_{0} \oplus \mathfrak{A}_{1} \oplus \mathfrak{A}_{2}$ by (35), the same unit $c=1 \oplus 0 \oplus 0$, and the same norm form (28)

$$
\begin{aligned}
N\left(a_{0}+b_{1}+c_{2}\right)= & N\left(a_{0}\right)+N\left(b_{1}\right)+N\left(c_{2}\right)+T\left(a_{0}^{\#}, b_{1}+c_{2}\right)+T\left(b_{1}^{\#}, c_{2}+a_{0}\right) \\
& +T\left(c_{2}^{\#}, a_{0}+b_{1}\right)+T\left(a_{0}, b_{1} \times c_{2}\right) \\
= & n(a)+\mu n(b)+\mu^{-1} n(c)-t(a b c)
\end{aligned}
$$

by (36), (38), (39). Thus $\Im(N, c) \cong \Im(\mathfrak{A}, \mu)$ as claimed.

Recall [3, p. 509] that one way of defining the algebra $\mathfrak{I}\left(\mathfrak{A}, \mu, u,{ }^{*}\right)$ given by the Second Tits Construction is the subalgebra $\mathfrak{S}\left(\mathfrak{I}(\mathfrak{A}, \mu),{ }^{*}\right)$ of $\mathfrak{\Im}(\mathfrak{A}, \mu)$ fixed by the semilinear involution

(40) $(a, b, u c) \rightarrow\left(a^{*}, c^{*}, u b^{*}\right)$.

THEOREM 9. If $\mathfrak{\Im}$ is a form of $\mathfrak{S}\left(\mathfrak{S}_{3}\right)$ which contains a subalgebra of the form $\mathfrak{S}\left(\mathfrak{U},{ }^{*}\right)$ for $\mathfrak{A}$ central simple associative of degree 3 over $\Omega$ with involution * of second kind having $\Phi$ as fixed field, then $\mathfrak{\Im}=\mathfrak{\Im}\left(\mathfrak{H}, \mu, u,{ }^{*}\right)$ for some $\mu \in \Omega$ and $u \in \mathfrak{S}\left(\mathfrak{H},{ }^{*}\right)$ with $n(u)=\mu \mu^{*}$.

Proof. We have $\Omega=\Phi+\lambda \Phi$ where $\lambda+\lambda^{*}=1$, and $\mathfrak{A}=\mathfrak{H}+\lambda \mathscr{H}=\Omega \otimes \mathfrak{H}$ for $\mathfrak{S}=\mathfrak{S}\left(\mathfrak{A},{ }^{*}\right)$. Thus $\mathfrak{I}_{\Omega}=\Omega \otimes \mathfrak{I}=\mathfrak{I}+\lambda \mathfrak{I}$ contains a subalgebra of the form $\mathfrak{A}^{+}$. By Theorem $8, \mathfrak{J}_{\Omega} \cong \mathfrak{I}(\mathfrak{A}, \mu)$ for some $\mu \in \Omega$. Note also that the involution * on $\Omega$ extends to an involution $* \otimes I$ on $\Omega \otimes \mathfrak{S}=\mathfrak{I}_{\Omega}$, which we will continue to call $*$ because it coincides with the original involution on $\mathfrak{A}=\Omega \otimes \mathfrak{H}$. We examine how this involution acts in $\mathfrak{I}_{\Omega}=\mathfrak{\Im}(\mathfrak{A}, \mu)=\mathfrak{A}_{0} \oplus \mathfrak{A}_{1} \oplus \mathfrak{A}_{2}$. We claim $\mathfrak{A}_{1}^{*} \subset \mathfrak{A}_{2}$, since if $a \times\left(b \times m_{1}\right)=-(a b) \times m_{1}$ for all $a, b \in \mathfrak{A}_{0}$ then (since the semilinear involution preserves generic norms and adjoints) $a^{*} \times\left(b^{*} \times m_{1}^{*}\right)=\left\{a \times\left(b \times m_{1}\right)\right\}^{*}=-\left\{(a b) \times m_{1}\right\}^{*}$ $=-(a b)^{*} \times m_{1}^{*}=-\left(b^{*} a^{*}\right) \times m_{1}^{*}$ (remember that $*$ reduces to the original involution 
on $\mathfrak{U}=\mathfrak{U}_{0}$ ); this holds for all $a^{*}, b^{*}$ in $\mathfrak{A}_{0}^{*}=\mathfrak{A}_{0}$, so $m_{1}^{*} \in \mathfrak{A}_{2}$ by (32). Similarly $\mathfrak{A}_{2}^{*} \subset \mathfrak{A}_{1}$. Thus $(0,1,0)^{*}=(0,0, u)$ and $(0,0,1)^{*}=(0, v, 0)$ for some $u, v \in \mathfrak{A}$. But using (28) we see $\mu^{-1} n(u)=N(0,0, u)=N\left((0,1,0)^{*}\right)=N(0,1,0)^{*}=\{\mu n(1)\}^{*}=\mu^{*}$, or

(41) $n(u)=\mu \mu^{*}$

and $\left((v u)^{*}, 0,0\right)=(v u, 0,0)^{*}=-\{(0, v, 0) \times(0,0, u)\}^{*}=-\left\{(0,0,1)^{*} \times(0,1,0)^{*}\right\}^{*}$ $=-(0,0,1) \times(0,1,0)=(1,0,0)$, so

(42) $v=u^{-1}$.

For arbitrary $(a, b, c)=(a, 0,0)-(b, 0,0) \times(0,1,0)-(c, 0,0) \times(0,0,1)$ in $\mathfrak{A}_{0} \oplus \mathfrak{A}_{1}$ $\oplus \mathfrak{A}_{2}$ we have $(a, b, c)^{*}=\left(a^{*}, 0,0\right)-\left(b^{*}, 0,0\right) \times(0,0, u)-\left(c^{*}, 0,0\right) \times\left(0, u^{-1}, 0\right)=$ $\left(a^{*}, c^{*} u^{-1}, u b^{*}\right)$, so the involution * takes the form

(43) $(a, b, c)^{*}=\left(a^{*}, c^{*} u^{-1}, u b^{*}\right)$.

Furthermore, since $(0,1,0)=(0,1,0)^{* *}=(0,0, u)^{*}=\left(0, u^{*} u^{-1}, 0\right)$ we see

(44) $u=u^{*} \in \mathfrak{S}\left(\mathfrak{A},{ }^{*}\right)$.

Once we know (44), we can rewrite (43) as (40): $(a, b, u c)^{*}=\left(a^{*}, c^{*}, u b^{*}\right)$. Thus $\mathfrak{\Im}=\mathfrak{S}\left(\mathfrak{J}_{\Omega},{ }^{*}\right) \cong \mathfrak{S}\left(\mathfrak{I}(\mathfrak{A}, \mu),{ }^{*}\right)$ where the involution on $\mathfrak{\Im}(\mathfrak{A}, \mu)$ is of the form $(40)$. But then the subalgebra of symmetric elements is isomorphic to $\Im\left(\mathfrak{A}, \mu, u,{ }^{*}\right)$, and $\mathfrak{I} \cong \mathfrak{E}\left(\mathfrak{I}(\mathfrak{A}, \mu),{ }^{*}\right) \cong \mathfrak{I}\left(\mathfrak{A}, \mu, u,{ }^{*}\right)$ as asserted by the theorem.

Putting the pieces together, we can sum up by

THEOREM 10. $\Im$ is a form of the split exceptional quadratic Jordan algebra $\mathfrak{S}\left(\mathfrak{E}_{3}\right)$ if and only if $\mathfrak{\Im}=\Im(\mathfrak{H}, \mu)$ or $\mathfrak{\Im}=\mathfrak{I}\left(\mathfrak{A}, \mu, u,{ }^{*}\right)$ is obtained by the First or Second Tits Construction.

Proof. By Lemma 6 and Theorems 8 and 9 , every form $\Im$ of $\mathfrak{S}_{(}\left(\mathfrak{C}_{3}\right)$ is either a $\mathfrak{I}(\mathfrak{A}, \mu)$ or a $\mathfrak{I}\left(\mathfrak{A}, \mu, u,{ }^{*}\right)$. Conversely, every $\mathfrak{I}\left(\mathfrak{A}, \mu, u,{ }^{*}\right)$ is a form of a $\mathfrak{I}(\mathfrak{A}, \mu)$ and every $\mathfrak{I}(\mathfrak{A}, \mu)$ is a form of $\Im\left(\Omega_{3}, \mu\right)$ since any central simple $\mathfrak{A}$ of degree 3 is a form of $\Omega_{3}$. Now over the algebraic closure $\mu^{-1}$ is a norm, and in general $\mathfrak{(}(\mathfrak{A}, \mu)$ $\cong \mathfrak{I}(\mathfrak{A}, \mu n(u))$ under $(a, b, c) \rightarrow\left(a, b u^{-1}, u c\right)$ since that map sends $(1,0,0)$ into $(1,0,0)$ and preserves the norm

$$
\begin{aligned}
\tilde{N}\left(a, b u^{-1}, u c\right) & =n(a)+\mu n(u) n\left(b u^{-1}\right)+\mu^{-1} n(u)^{-1} n(u c)-t\left(a\left(b u^{-1}\right)(u c)\right) \\
& =n(a)+\mu n(b)+\mu^{-1} n(c)-t(a b c)=N(a, b, c),
\end{aligned}
$$

so all $\Im(\mathfrak{A}, \mu)$ and $\Im\left(\mathfrak{A}, \mu, u,{ }^{*}\right)$ are forms of the algebra $\Im\left(\Omega_{3}, 1\right)$. In particular this holds for $\mathfrak{S}_{\mathfrak{S}}\left(\mathfrak{S}_{3}\right)$, so $\mathfrak{\Im}\left(\Omega_{3}, 1\right) \cong \mathfrak{S}\left(\mathfrak{S}_{3}\right)$, and all $\mathfrak{\Im}(\mathfrak{A}, \mu)$ and $\mathfrak{I}\left(\mathfrak{H}, \mu, u,{ }^{*}\right)$ are forms of $\mathfrak{S}\left(\mathfrak{S}_{3}\right)$.

\section{REFERENCES}

1. N. Jacobson, Structure and representations of Jordan algebras, Amer. Math. Soc. Colloq. Publ., vol. 39, Amer. Math. Soc., Providence, R. I., 1968.

2. - Associative algebras with involution and Jordan algebras, Nederl. Akad. Wetensch. Proc. Ser. A. 69 (1966), 202-212=Indag. Math. 28 (1966), 202-212. MR 33 \#5686.

3. K. McCrimmon, The Freudenthal-Springer-Tits constructions of exceptional Jordan algebras, Trans. Amer. Math. Soc. 139 (1969), 495-510. 
4. K. McCrimmon, A general theory of Jordan rings, Proc. Nat. Acad. Sci. U.S.A. 56 (1966), 1072-1079, MR 34 \#2643.

5. - Generically algebraic algebras, Trans. Amer. Math. Soc. 127 (1967), 527-551. MR 35 \#1644.

6. - Jordan algebras with interconnected idempotents, Proc. Amer. Math. Soc. 19 (1968), 1327-1336. MR 38 \#203.

7. — Macdonald's theorem for quadratic Jordan algebras, (to appear).

8. - A proof of Schafer's conjecture for infinite-dimensional forms admitting composition, J. Algebra 5 (1967), 72-83. MR 34 \#4318.

9. - The radical of a Jordan algebra, Proc. Nat. Acad. Sci. U.S.A. 62 (1969), 671-678. 10. N. Jacobson, Lie algebras, Interscience, New York, 1962. MR 26 \#1345.

11. - Lectures in abstract algebra. Vol. III, Van Nostrand, Princeton, N. J., 1964. MR 30 \#3087.

UNIVERSITY OF VIRGINIA,

Charlottesville, Virginia 